\title{
Mathematical Analysis of a General Two-Patch Model of Tuberculosis Disease with Lost Sight Individuals
}

\author{
Abdias Laohombé, ${ }^{1}$ Isabelle Ngningone Eya, ${ }^{2}$ Jean Jules Tewa, ${ }^{2}$ Alassane Bah, ${ }^{3}$ \\ Samuel Bowong, ${ }^{4}$ and Suares Clovis Oukouomi Noutchie ${ }^{5}$ \\ ${ }^{1}$ Department of Mathematics, Faculty of Science, University of Yaounde I, Yaounde 8390, Cameroon \\ ${ }^{2}$ Department of Mathematics and Physics, National Advanced School of Engineering, University of Yaounde I, \\ UMMISCO, Team Project GRIMCAPE, LIRIMA, Yaounde 8390, Cameroon \\ ${ }^{3}$ Cheikh Anta Diop University, National Advanced School of Engineering, UMMISCO, 5085 Dakar, Senegal \\ ${ }^{4}$ Department of Mathematics and Computer Science, Faculty of Science, University of Douala, \\ UMMISCO, Team Project GRIMCAPE, LIRIMA, Douala 24157, Cameroon \\ ${ }^{5}$ MaSIM Focus Area, North-West University, Mafikeng Campus, Mafikeng 2735, South Africa \\ Correspondence should be addressed to Suares Clovis Oukouomi Noutchie; 23238917@nwu.ac.za
}

Received 30 May 2014; Accepted 25 June 2014; Published 20 July 2014

Academic Editor: Abdon Atangana

Copyright (C) 2014 Abdias Laohombé et al. This is an open access article distributed under the Creative Commons Attribution License, which permits unrestricted use, distribution, and reproduction in any medium, provided the original work is properly cited.

A two-patch model, $S E_{i 1}, \ldots, E_{i n} I_{i} L_{i}, i=1,2$, is used to analyze the spread of tuberculosis, with an arbitrary number $n$ of latently infected compartments in each patch. A fraction of infectious individuals that begun their treatment will not return to the hospital for the examination of sputum. This fact usually occurs in sub-Saharan Africa, due to many reasons. The model incorporates migrations from one patch to another. The existence and uniqueness of the associated equilibria are discussed. A Lyapunov function is used to show that when the basic reproduction ratio is less than one, the disease-free equilibrium is globally and asymptotically stable. When it is greater than one, there exists at least one endemic equilibrium. The local stability of endemic equilibria can be illustrated using numerical simulations. Numerical simulation results are provided to illustrate the theoretical results and analyze the influence of lost sight individuals.

\section{Introduction}

For a given system, the focus in qualitative mathematical epidemiology is the long-term dynamics. The simplest possible attractor is a globally and asymptotically stable equilibrium. Equilibrium can be shown to be globally and asymptotically stable, using Poincaré-Bendixson theory [1], Bendixson's negative criterion [2,3], or the generalized version of Dulac [4]. Another method of Li and Muldowney [5-7] for demonstrating global stability in $n$ dimensions has been developed more recently, with applications in three $[8,9]$ and four dimensions $[10,11]$. For higher-dimensional systems, the theory of quadratic forms [12] or Lyapunov's method can be used $[13,14]$. Lyapunov's method requires finding a function $V$ such that the flow always crosses the level sets from higher values of $V$ to lower values. When such a function can be found, then any isolated minimum of the function is a stable equilibrium of the flow. In this paper, the stability of a $2 n+6$-dimensions system is investigated using Lyapunov-LaSalle functions and quadratic forms. The function $V=\sum_{i=1}^{n} a_{i}\left(x_{i}-x_{i}^{*} \ln x_{i}\right)$ has a long story in epidemiology [15-20]. Volterra himself originally discovered this function, although he did not use the vocabulary and the theory of Lyapunov functions. Since epidemic models are Lotka-Volterra-like models, the pertinence of this function is not surprising.

The issue of modeling tuberculosis motivates the model studied in this paper. It is an extension with two patches and $n$ latent classes of the SEIL model in [21], where $L$ denotes the lost sight individuals. These lost sight individuals usually occur in sub-Saharan Africa. For example, 
according to the Cameroonian National Program of Fight against Tuberculosis, about $10 \%$ of infectious individuals that begun their therapy treatment lost sight. Therefore, this fact cannot be neglected in the modeling of TB. By allowing for an arbitrary number of latently infected compartments, the model allows for the approximation of a wide class of distributions of latency durations. This is of particular importance for tuberculosis since latency may last for years or even decades.

In the model given here, there are migrations between people of two patches, who have the same epidemiological characteristics. We introduce a direct transfer from the class of susceptible individuals toward the compartment of infectious individuals in each patch. The reason is that there are some individuals with bad immune effector protection, due to many reasons. We also incorporate a transfer from the infectious class to the exposed class to take into account the fact that some infectious individuals apparently recover but actually harbor TB bacteria. Let us give now the outlines of the paper. In Section 2, the model is constructed; the variables and parameters of the model are explained. In Section 3, the mathematical properties of the model are given. We present the computation thresholds as basic reproduction numbers, which are bifurcation parameters $R_{0}^{i}$, using the same method as in [22]. The equilibria of the model are computed: equilibrium without disease, endemic equilibria, or coexistence equilibria. The stability of each equilibrium is investigated, using the bifurcation parameters $R_{0}^{i}$. In Section 4, numerical simulations are done to illustrate the results. In Section 5, we give the conclusion.

\section{Model Construction}

The model consisted of two patches. Each patch denotes a given population and the two populations are very much closed. Based on epidemiological status, the population of a given patch $i(i=1,2)$ is divided into $n+3$ classes: susceptible $\left(S_{i}\right)$, latently infected $\left(E_{i}\right)$, infectious $\left(I_{i}\right)$, and lost sight $\left(L_{i}\right)$. All recruitments in a given patch $i$ are into the susceptible class and occur at a constant rate $\Lambda_{i}$. The rate constant for nondisease related death is $\mu_{i}$; thus $1 / \mu_{i}$ is the average lifetime. Infectious and lost sight classes of a patch $i$ have addition death rates due to the disease with rates constants $d_{i}$ and $l_{i}$, respectively. Since we do not know if lost sight class is recovered, died, or is still infectious, we assume that a fraction $\delta_{i}$ of them is still infectious and can transmit disease to susceptible class. Transmission of Mycobacterium tuberculosis occurs following adequate contacts between susceptible and infectious or lost sight classes that continue to have disease. We assume that infected individuals are not infectious and thus are not capable of transmitting bacteria. We use the universal incidence expressions $\beta_{i} S_{i} I_{i} / N_{i}$ and $\beta_{i} \delta_{i} S_{i} L_{i} / N_{i}$ to indicate successful transmission of $M$. tuberculosis due to nonlinear contacts dynamics in the population by infectious and lost sight classes, respectively. A fraction $p_{i}$ of the newly infected individuals is assumed to undergo fast progression directly to the infectious class, while the remainder are latently infected and enter the latent class. Once latently infected with M. tuberculosis, an individual will remain so for life unless reactivation occurs. To account for treatment, we define $r_{i j} E_{i}$, with $i=1,2$ and $j=1, \ldots, n$, as the fraction of infected individuals receiving effective chemoprophylaxis, and $\gamma_{i}$ as the rate of effective per capita therapy. We assume that chemoprophylaxis of latently infected individuals $E_{i j}$ reduces their reactivation at rate $r_{i j}$. Thus, a fraction (1$\left.r_{i j}\right) E_{i}$ of infected individuals who do not receive effective chemoprophylaxis becomes infectious with a rate constant $k_{i}$, so that $1 / k_{i}$ represents the average latent period. Thus, individuals leave the class $E_{i j}$ to $I_{i}$ at rate $k_{i}\left(1-r_{i j}\right)$. After receiving an effective therapy, infectious individuals can spontaneously recover from the disease with a rate constant $\gamma_{i}$, entering the infected class $E_{i n}$. A fraction $s_{i}\left(1-r_{i j}\right) I_{i}$ of infectious individuals that began their treatment will not return to the hospital for the examination of sputum. After some time, some of them will return to the hospital with the disease at a constant rate $\alpha_{i}$. This can be the situation in many African countries or refugees camps in Africa or elsewhere.

The transfer diagram of the model is given by Figure 1 .

This yields the following set of differential equations:

$$
\begin{aligned}
& \frac{d S_{1}}{d t}=\Lambda_{1}-\left(\mu_{1}+a_{1}\right) S_{1} \\
& -\beta_{1} \frac{\left(I_{1}+\delta_{1} L_{1}\right) S_{1}}{N_{1}}+a_{2} S_{2} \\
& \frac{d E_{11}}{d t}=\beta_{1}\left(1-p_{1}\right) \frac{\left(I_{1}+\delta_{1} L_{1}\right) S_{1}}{N_{1}} \\
& +b_{21} E_{21}-\left(\mu_{1}+b_{11}+\left(1-r_{11}\right) k_{11}\right) E_{11} \text {, } \\
& \frac{d E_{12}}{d t}=\left(1-r_{11}\right) k_{11} E_{11}+b_{22} E_{22} \\
& -\left(\mu_{1}+b_{12}+\left(1-r_{12}\right) k_{12}\right) E_{12} \\
& \frac{d E_{1 n}}{d t}=\left(1-r_{1, n-1}\right) k_{1, n-1} E_{1, n-1}+\gamma_{1} I_{1} \\
& +b_{2 n} E_{2 n}-\left(\mu_{1}+b_{1 n}+\left(1-r_{1 n}\right) k_{1 n}\right) E_{1 n}, \\
& \frac{d I_{1}}{d t}=\beta_{1} p_{1} \frac{\left(I_{1}+\delta_{1} L_{1}\right) S_{1}}{N_{1}}+\left(1-r_{1 n}\right) k_{1 n} E_{1 n} \\
& +\alpha_{1} L_{1}+c_{2} I_{2}-\left(\mu_{1}+d_{1}+\gamma_{1}+\left(1-v_{1}\right) s_{1}+c_{1}\right) I_{1} \text {, } \\
& \frac{d L_{1}}{d t}=\left(1-v_{1}\right) s_{1} I_{1}+f_{2} L_{2} \\
& -\left(\mu_{1}+l_{1}+\alpha_{1}+f_{1}\right) L_{1} \\
& \frac{d S_{2}}{d t}=\Lambda_{2}-\left(\mu_{2}+a_{2}\right) S_{2} \\
& -\beta_{2} \frac{\left(I_{2}+\delta_{2} L_{2}\right) S_{2}}{N_{2}}+a_{1} S_{1}
\end{aligned}
$$




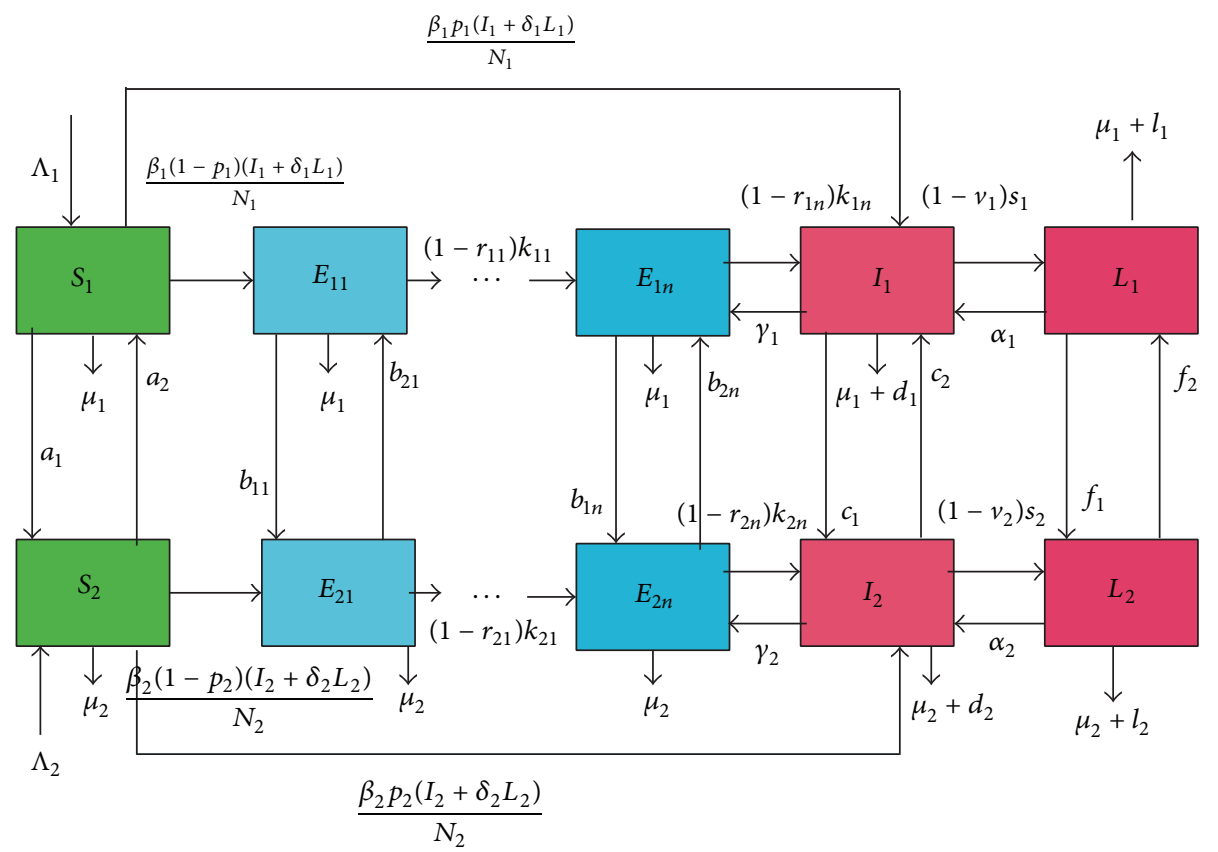

FIGURE 1: Flow chart of a two-patch model of tuberculosis with lost sight individuals in sub-Saharan Africa, with migrations at rate $a_{i}$ between susceptible individuals of two populations, at rate $b_{i j}$ between latently infected individuals of two populations, at rate $c_{i}$ between infectious individuals in care centre, and at rate $f_{i}$ for infectious lost sight individuals. An individual of a given population (patch) has contacts only with individuals of the same population.

$$
\begin{gathered}
\frac{d E_{21}}{d t}=\beta_{2}\left(1-p_{2}\right) \frac{\left(I_{2}+\delta_{2} L_{2}\right) S_{2}}{N_{2}} \\
+b_{11} E_{11}-\left(\mu_{2}+b_{21}+\left(1-r_{21}\right) k_{21}\right) E_{21} \\
\frac{d E_{22}}{d t}=\left(1-r_{21}\right) k_{21} E_{21}+b_{12} E_{12} \\
-\left(\mu_{2}+b_{22}+\left(1-r_{22}\right) k_{22}\right) E_{22} \\
\vdots \\
\begin{array}{c}
\frac{d E_{2 n}}{d t}= \\
+\gamma_{2} I_{2}+b_{1 n} E_{1 n} \\
-\left(\mu_{2}+b_{2 n}+\left(1-r_{2 n}\right) k_{2 n}\right) E_{2 n} \\
\quad\left(I_{2}+\delta_{2} L_{2}\right) S_{2} \\
N_{2} \\
+\left(1-r_{2 n}\right) k_{2 n} E_{2 n}+\alpha_{2} L_{2}+c_{1} I_{1} \\
-\left(\mu_{2}+d_{2}+\gamma_{2}+\left(1-v_{2}\right) s_{2}+c_{2}\right) I_{2} \\
\frac{d I_{2}}{d t}=\left(1-v_{2}\right) s_{2} I_{2}+f_{1} L_{1}-\left(\mu_{2}+l_{2}+\alpha_{2}+f_{2}\right) L_{2}
\end{array}
\end{gathered}
$$

where $N_{1}=S_{1}+E_{11}+\cdots+E_{1 n}+I_{1}+L_{1}$ and $N_{2}=S_{2}+E_{21}+$ $\cdots+E_{2 n}+I_{2}+L_{2}$.

System (1) can also be written as

$$
\begin{aligned}
& \frac{d x_{1}}{d t}=\Lambda_{1}-\left(\mu_{1}+a_{1}\right) x_{1}-\beta_{1} \frac{x_{1}}{N_{1}}\left\langle e_{n+1}^{n+2}+\frac{\delta_{1} e_{n+2}^{n+2}}{Y_{1}}\right\rangle, \\
& \frac{d x_{1}}{d t}=\Lambda_{2}-\left(\mu_{2}+a_{2}\right) x_{2}-\beta_{2} \frac{x_{2}}{N_{2}}\left\langle e_{n+1}^{n+2}+\frac{\delta_{2} e_{n+2}^{n+2}}{Y_{2}}\right\rangle, \\
& \frac{d Y_{1}}{d t}=\beta_{1} \frac{x_{1}}{N_{1}}\left\langle e_{n+1}^{n+2}+\frac{e_{n+2}^{n+2}}{Y_{1}}\right\rangle Q_{1}+A_{11} Y_{1}+A_{12} Y_{2}, \\
& \frac{d Y_{2}}{d t}=\beta_{2} \frac{x_{2}}{N_{2}}\left\langle e_{n+1}^{n+2}+\frac{e_{n+2}^{n+2}}{Y_{1}}\right\rangle Q_{2}+A_{21} Y_{1}+A_{22} Y_{2},
\end{aligned}
$$

where $\langle\cdot / \cdot\rangle$ is the scalar usual product in $I R_{+}^{n+2}, Q_{1}=((1-$ $\left.\left.p_{1}\right), 0, \ldots, 0, p_{1}\right)^{T}, Q_{2}=\left(\left(1-p_{2}\right), 0, \ldots, 0, p_{2}\right)^{T}, x=\left(x_{1}, x_{2}\right)^{T}$, $Y_{1}=\left(E_{11}, \ldots, E_{1 n}, I_{1}, L_{1}\right)^{T}=\left(y_{11}, \ldots, y_{1 n}, y_{1, n+1}, y_{1, n+2}\right)^{T}$, $Y_{1}=\left(E_{21}, \ldots, E_{2 n}, I_{2}, L_{2}\right)^{T}=\left(y_{21}, \ldots, y_{2 n}, y_{2, n+1}, y_{2, n+2}\right)^{T}$, $\left(e_{i}^{n+2}\right)$ is the canonical basis of $I R_{+}^{n+2}$, the matrices $A_{11}$ and $A_{22}$ are Metzler stable [23], and $A_{12}$ and $A_{21}$ are Metzler stable and given, respectively, by 


$$
\begin{aligned}
& A_{11}=\left(\begin{array}{ccccccc}
-m_{11} & 0 & \ldots & 0 & 0 & 0 & 0 \\
\left(1-r_{11}\right) k_{11} & -m_{12} & 0 & \ldots & 0 & 0 & 0 \\
\cdots & \ldots & \ldots & \ldots & \ldots & \cdots & \ldots \\
0 & \ldots & 0 & \left(1-r_{1, n-1}\right) k_{1, n-1} & -m_{1 n} & \gamma_{1} & 0 \\
0 & 0 & \ldots & 0 & \left(1-r_{1, n}\right) k_{1, n} & -m_{I_{1}} & \alpha_{1} \\
0 & 0 & 0 & \ldots & 0 & \left(1-v_{1}\right) s_{1} & -m_{L_{1}}
\end{array}\right), \\
& A_{22}=\left(\begin{array}{ccccccc}
-m_{21} & 0 & \cdots & 0 & 0 & 0 & 0 \\
\left(1-r_{21}\right) k_{21} & -m_{22} & 0 & \cdots & 0 & 0 & 0 \\
\cdots & \cdots & \cdots & \cdots & \cdots & \cdots & \cdots \\
0 & \ldots & 0 & \left(1-r_{2, n-1}\right) k_{2, n-1} & -m_{2 n} & \gamma_{2} & 0 \\
0 & 0 & \cdots & 0 & \left(1-r_{2, n}\right) k_{2, n} & -m_{I_{2}} & \alpha_{2} \\
0 & 0 & 0 & \cdots & 0 & \left(1-v_{2}\right) s_{2} & -m_{L_{2}}
\end{array}\right) \text {, } \\
& A_{12}=\left(\begin{array}{cccccc}
b_{21} & 0 & 0 & \ldots & 0 & 0 \\
0 & b_{22} & 0 & \ldots & 0 & 0 \\
0 & \ldots & 0 & b_{2 n} & 0 & 0 \\
0 & \ldots & 0 & 0 & c_{2} & 0 \\
0 & \ldots & 0 & 0 & 0 & f_{2}
\end{array}\right) \\
& A_{21}=\left(\begin{array}{cccccc}
b_{11} & 0 & 0 & \ldots & 0 & 0 \\
0 & b_{12} & 0 & \ldots & 0 & 0 \\
0 & \ldots & 0 & b_{1 n} & 0 & 0 \\
0 & \ldots & 0 & 0 & c_{1} & 0 \\
0 & \ldots & 0 & 0 & 0 & f_{1}
\end{array}\right)
\end{aligned}
$$

where $m_{i 1}=\left(\mu_{i}+b_{i 1}+\left(1-r_{i 1}\right) k_{i 1}\right), m_{i n}=\left(\mu_{i}+b_{i n}+\left(1-r_{i n}\right) k_{i n}\right)$, $m_{I_{i}}=\left(\mu_{i}+d_{i}+\gamma_{i}+\left(1-v_{i}\right) s_{i}+c_{i}\right)$, and $m_{L_{i}}=\left(\mu_{i}+l_{i}+\alpha_{i}+f_{i}\right)$, $i=1,2$.

Let us give another expression of system (1). System (2) can be written as

$$
\begin{gathered}
\frac{d x(t)}{d t}=\Lambda+D_{x} x-\sum_{i=1}^{2}\left\langle\frac{B_{i}}{y(t)}\right\rangle \frac{\left\langle e_{i}^{2} / x(t)\right\rangle}{N_{i}} e_{i}^{2}, \\
\frac{d y(t)}{d t}=\sum_{i=1}^{2}\left\langle\frac{B_{i}}{y(t)}\right\rangle \frac{\left\langle e_{i}^{2} / x(t)\right\rangle}{N_{i}} K_{i}+A_{y} y,
\end{gathered}
$$

where $\langle\cdot / \cdot\rangle$ is now the usual scalar product in $I R^{2 n+4}$, $x=\left(x_{1}, x_{2}\right)^{T}, e_{1}^{2}=(1,0)^{T}, e_{2}^{2}=(0,1)^{T}, \Lambda=\left(\Lambda_{1}, \Lambda_{2}\right)^{T}$, $y=\left(E_{11}, \ldots, E_{1 n}, I_{1}, L_{1}, E_{21}, \ldots, E_{2 n}, I_{2}, L_{2}\right)^{T}, D_{x}=$ $\left(\begin{array}{cc}-\left(\mu_{1}+a_{1}\right) & a_{2} \\ a_{1} & -\left(\mu_{2}+a_{2}\right)\end{array}\right), B_{1}=(\underbrace{0, \ldots, 0}_{n}, \beta_{1}, \beta_{1} \delta_{1}, \underbrace{0, \ldots, 0}_{n+2})^{T}$, $B_{2}=(\underbrace{0, \ldots, 0}_{n+2}, \underbrace{0, \ldots, 0}_{n}, \beta_{2}, \beta_{2} \delta_{2})^{T}, K_{1}=(1-$ $p_{1}, \underbrace{0, \ldots, 0}_{n-1}, p_{1}, 0, \underbrace{0, \ldots, 0}_{n+2})^{T}, \quad K_{2}=(\underbrace{0, \ldots, 0}_{n+2}, 1-$ $p_{2}, \underbrace{0, \ldots, 0}_{n-1}, p_{2}, 0)^{T}$, and $A_{y}=\left(\begin{array}{ll}A_{11} & A_{12} \\ A_{21} & A_{22}\end{array}\right)$.

\section{Mathematical Properties}

3.1. Positivity of the Solutions. Since the variables considered here are nonnegative quantities, we have to be sure that their values are always nonnegative.
Theorem 1. The nonnegative orthant $I R_{+}^{2 n+6}$ is positively invariant by (1). This means that every trajectory, which begins in the positive orthant, will stay inside.

Proof. System (4) can be written in the following form:

$$
\begin{gathered}
\frac{d x(t)}{d t}=\Lambda+D_{x} x-\sum_{i=1}^{2}\left\langle\frac{B_{i}}{y(t)}\right\rangle \frac{\left\langle e_{i}^{2} / x(t)\right\rangle}{N_{i}} e_{i}^{2}, \\
\frac{d y(t)}{d t}=\left(\sum_{i=1}^{2} B_{i}^{T} \frac{\left\langle e_{i}^{2} / x(t)\right\rangle}{N_{i}} K_{i}+A_{y}\right) y(t) .
\end{gathered}
$$

Since $x(t) \geq 0$ and $N_{i}(t) \geq 0$, the matrix $\left(\sum_{i=1}^{2} B_{i}^{T}\left(\left\langle e_{i}^{2} / x(t)\right\rangle / N_{i}\right) K_{i}+A_{y}\right)$ is Metzler. It is well known that linear Metzler system lets the nonnegative orthant invariant [23]. Moreover $d x(t) / d t \leq \Lambda+D_{x} x$ and $D_{x}$ is a Metzler matrix. These prove the positive invariance of the nonnegative orthant $I R_{+}^{2 n+6}$ by (1).

\subsection{Boundedness of the Trajectories}

Lemma 2. The simplex $\left\{\left(S_{1}, E_{11}, \ldots, E_{1 n}, I_{1}, L_{1}, S_{2}, E_{21}, \ldots\right.\right.$, $\left.E_{2 n}, I_{2}, L_{2}\right) \in I R_{+}^{2 n+6} / S_{1}+E_{11}+\cdots E_{1 n}+I_{1}+L_{1}+S_{2}+E_{21}+$ $\left.\cdots E_{2 n}+I_{2}+L_{2} \leq\left(\left(\Lambda_{1}+\Lambda_{2}\right) / \mu\right)+\varepsilon\right\}$, is a compact forward and absorbing set for (1).

Proof. From system (1), if $N(t)=N_{1}(t)+N_{2}(t)$ denotes the entire population of (1), one has $N(t)=S_{1}+E_{11}+\cdots+E_{1 n}+$ $I_{1}+L_{1}+S_{2}+E_{21}+\cdots+E_{2 n}+I_{2}+L_{2}$. 
Then, the dynamics of $N(t)$ at any time $t$ is given by

$$
\begin{aligned}
\frac{d N(t)}{d t}= & \Lambda_{1}+\Lambda_{2}-\mu_{1} N_{1}(t)-\mu_{2} N_{2}(t)-d_{1} I_{1} \\
& -l_{1} L_{1}-d_{2} I_{2}-l_{2} L_{2} .
\end{aligned}
$$

Thus, $d N(t) / d t \leq \Lambda_{1}+\Lambda_{2}-\mu N(t)$, where $\mu=\min \left(\mu_{1}, \mu_{2}\right)$. It follows that $\operatorname{limSup}_{t \rightarrow+\infty} N(t)=\left(\Lambda_{1}+\Lambda_{2}\right) / \mu$.

In the simplex $\Gamma_{\varepsilon}$, (1) is mathematically well posed. The following lemma also holds.

Lemma 3. The simplex $\gamma_{\varepsilon}=\left\{(x, y) \in \Gamma_{\varepsilon} / x \leq x^{*}\right\}$ is a compact forward invariant set for (1).

3.3. Local Stability of the Disease-Free Equilibrium. Many epidemiological models have a threshold condition which can determine whether an infection will be eliminated from the population or become endemic. The basic reproduction number, $R_{0}$, is defined as the average number of secondary infections produced by an infected individual in a completely susceptible population [16]. As discussed in [24, 25], $R_{0}$ is a simply normalized bifurcation (transcritical) parameter for epidemiological models, such that $R_{0}$ implies that the endemic steady state is stable (i.e., the infection persists), and $R_{0}$ implies that the uninfected steady state is stable (i.e., the infection can be eliminated from the population).

Equation (1) has a disease-free equilibrium given by $K_{2}=(S_{1}^{*}, \underbrace{0, \ldots, 0}_{n+2}, S_{2}^{*}, \underbrace{0, \ldots, 0}_{n+2})^{T}$ which always exists in the nonnegative orthant $I R_{+}^{2 n+6}$. The explicit expressions of $S_{1}^{*}$ and $S_{2}^{*}$ are

$$
S_{1}^{*}=\frac{\left(\mu_{2}+a_{2}\right) \Lambda_{1}+a_{2} \Lambda_{2}}{\mu_{1} \mu_{2}+\mu_{1} a_{2}+\mu_{2} a_{1}}, \quad S_{2}^{*}=\frac{\left(\mu_{1}+a_{1}\right) \Lambda_{2}+a_{1} \Lambda_{1}}{\mu_{1} \mu_{2}+\mu_{1} a_{2}+\mu_{2} a_{1}} .
$$

The disease-free equilibrium can also be denoted by $\left(x^{*}, 0\right)$ since it is the solution of equation $\Lambda+D_{x} x^{*}=0$ of the compact system (5).

Lemma 4. Using the same method as in [17], the basic reproduction ratio of $(1)$ is $R_{0}=\rho\left(K_{1} B_{1}^{T}\left(-A_{y}\right)^{-1}+K_{2} B_{2}^{T}\left(-A_{y}\right)^{-1}\right)$, where $\rho$ denotes the spectral radius.

Proof. The expressions, which are coming from the other compartment, due to contamination, are given by the following matrix:

$$
\begin{aligned}
F & =\frac{\partial}{\partial y}\left(\sum_{i=1}^{2}\left\langle\frac{B_{i}}{y(t)}\right\rangle \frac{\left\langle e_{i}^{2} / x(t)\right\rangle}{N_{i}} K_{i}\right)_{\left(x^{*}, 0\right)} \\
& =\sum_{i=1}^{2} \frac{\left\langle e_{i}^{2} / x^{*}\right\rangle}{N_{i}^{*}} K_{i} B_{i}^{T} .
\end{aligned}
$$

The expressions, which are coming from the other compartment, due to reasons different from contamination, are given by $V=-A_{y}$. The next generation matrix, since $\left\langle e_{i}^{2} / x^{*}\right\rangle=$ $N_{i}^{*}$, is

$$
\begin{aligned}
F V^{-1} & =\left(\sum_{i=1}^{2} \frac{\left\langle e_{i}^{2} / x^{*}\right\rangle}{N_{i}^{*}} K_{i} B_{i}^{T}\right)\left(-A_{y}\right)^{-1} \\
& =\left(\sum_{i=1}^{2} K_{i} B_{i}^{T}\right)\left(-A_{y}\right)^{-1} .
\end{aligned}
$$

We can observe that, since $R_{0}$ is the largest eigenvalue of the next generation matrix,

$$
R_{0}=\rho\left(F V^{-1}\right)=\rho\left(K_{1} B_{1}^{T}\left(-A_{y}\right)^{-1}+K_{2} B_{2}^{T}\left(-A_{y}\right)^{-1}\right),
$$

where $\rho$ denotes the spectral radius.

Consequently, from Theorem 2 of [22], the following lemma holds.

Lemma 5. The disease-free equilibrium of (1) is locally and asymptotically stable whenever $R_{0} \leq 1$ and unstable if $R_{0}>1$.

Remark 6. This lemma shows that if $R_{0} \leq 1$, a small flow of infectious individuals will not generate large outbreaks of the disease. To control the disease independently of the initial total number of infectious individuals, a global asymptotic stability property has to be established for the DFE when $R_{0} \leq 1$.

3.4. Global Stability of the Disease-Free Equilibrium. The following result helps to determine the stability and is related to LaSalle's principle [13]. Consider the differential equation

$$
\begin{gathered}
\frac{d x(t)}{d t}=X(x(t)), \\
X\left(x_{0}\right)=0,
\end{gathered}
$$

where $X$ is a $C^{1}$ function defined on an open set of $I R^{n}$ containing the closure $\bar{\Omega}$ of a positively invariant set $\Omega$ such that the equilibrium $x_{0}$ is in $\bar{\Omega}$. The following lemma holds.

Lemma 7. We assume that system (11) is point dissipative [1] on $\Omega$. In other words there exists a compact set $K \subset \Omega$ such that, for any $y \in \Omega$, there exists a time $t\left(y_{0}\right)>0$ such that, for any time $t>t\left(y_{0}\right)$, the trajectory with initial condition $y_{0}$ is in the interior of $K$. If there exists a $C^{1}$ function $V \geq 0$ defined on $\bar{\Omega}$, then

(1) $d V(t) / d t=\langle\delta V(x) / X(x)\rangle \leq 0$, for all $x \in \Omega$;

(2) the greatest invariant set $L$ contained in $E=\{x \in$ $\bar{\Omega}, d V(t) / d t=0\}$ is contained in a positively invariant set on which the restriction of system (11) is globally and asymptotically stable on $L$ at $x_{0}$. 
Then, $x_{0}$ is a globally and asymptotically stable equilibrium of system (11) on $\Omega$.

Theorem 8. When $R_{0} \leq 1$ (this implies $R_{0}^{1} \leq 1$ and $R_{0}^{2} \leq$ 1 , where $R_{0}^{i}$ is the basic reproduction number for the patch $i)$, the disease-free equilibrium (DFE), when it is unique, is globally and asymptotically stable in $\Gamma_{\varepsilon}$, since it is the unique equilibrium. This implies the global asymptotic stability of the DFE on the nonnegative orthant $I R_{+}^{2 n+6}$; that is, the disease dies out in both two populations.

Remark 9. At least one endemic equilibrium can exist and coexist with the disease-free equilibrium when $R_{0} \leq 1$. In this case the DFE cannot be globally asymptotically stable.

Proof. Following the same method as in [23], system (4) can be written in a pseudotriangular form as

$$
\begin{gathered}
\frac{d x(t)}{d t}=A_{1}(x, y)\left(x-x^{*}\right)+A_{12}(x, y) y=f(x, y), \\
\frac{d y(t)}{d t}=A_{2}(x, y) y=g(x, y),
\end{gathered}
$$

where $f\left(x^{*}, 0\right)=\Lambda+D_{x} x^{*}=0, g\left(x^{*}, 0\right)=0, A_{1}(x, y)=D_{x} \epsilon$ $I R^{2 x 2}, A_{12}(x, y)=-\sum_{i=1}^{2}\left(\left\langle e_{i}^{2} / x(t)\right\rangle / N_{i}\right) e_{i}^{2} B_{i}^{T} \in I R^{2 x(2 n+4)}$, and $A_{2}(x, y)=\sum_{i=1}^{2}\left(\left\langle e_{i}^{2} / x(t)\right\rangle / N_{i}\right) K_{i} B_{i}^{T}+A_{y} \in I R^{(2 n+4) x(2 n+4)}$. The Jacobian matrix of system (4) at disease-free equilibrium $\left(x^{*}, 0\right)$ is $A_{2}(x, y)=\sum_{i=1}^{2}\left(\left\langle e_{i}^{2} / x(t)\right\rangle / N_{i}\right) K_{i} B_{i}^{T}+A_{y} \in$ $I R^{(2 n+4) x(2 n+4)}$. The Jacobian matrix of system $(4)$ at diseasefree equilibrium $\left(x^{*}, 0\right)$ is $J=\left(\begin{array}{cc}A_{1}\left(x^{*}, 0\right) & A_{12}\left(x^{*}, 0\right) \\ 0 & A_{2}\left(x^{*}, 0\right)\end{array}\right)=$ $\left(\begin{array}{cc}D_{x} & -\sum_{i=1}^{2} e_{i}^{2} B_{i}^{T} \\ 0 & \sum_{i=1}^{2} K_{i} B_{i}^{T}+A_{y}\end{array}\right)$. The matrix $D_{x}$ is clearly a Metzler stable matrix and, using a result in [8], there exists a vector $u=$ $\left(u_{1}, u_{2}\right)>0$ such that $D_{x} u \leq 0$. The matrix $\bar{A}_{2}=\sum_{i=1}^{2} K_{i} B_{i}^{T}+$ $A_{y}$ is a Metzler and irreducible matrix, which is stable if $R_{0} \leq 1$. With this condition satisfied and using the same previous result in [22], the stability modulus $\alpha\left(\bar{A}_{2}\right)$ of the matrix is in the spectrum of $\bar{A}_{2}$ (Perron-Frobenius theorem, proof of Lemma 2) and there exists a vector $c>0 \in I R^{2 n+4}$ such that $c^{T} \bar{A}_{2}=\alpha\left(\bar{A}_{2}\right) c^{T}$, with $\alpha\left(\bar{A}_{2}\right) \leq 0$. Let us show the global stability of the DFE.

System (12) is in pseudotriangular form since the matrix $A_{2}(x, y)$ depends on $(x, y)$. There are many results for the stability of triangular systems [21-25]. Using LaSalle's principle [13] one can obtain attractivity of equilibrium. But, for nonlinear systems, attractivity does not generally implies stability. We can use now the result of Lemma 7. Let us consider the following candidate Lyapunov function:

$$
V_{\mathrm{DFE}}(x(t), y(t))=\left\langle\frac{(0, c)}{(x(t), y(t))^{T}}\right\rangle .
$$

The function $V_{\mathrm{DFE}}(x(t), y(t))$ is positive and it is time derivative along the trajectories of (4) which gives

$$
\begin{aligned}
\frac{d V(x(t), y(t))}{d t} & =\left(\sum_{i=1}^{2}\left\langle\frac{B_{i}}{y(t)}\right\rangle \frac{\left\langle e_{i}^{2} / x(t)\right\rangle}{N_{i}} K_{i}+A_{y} y\right) c \\
& \leq c^{T}\left(\sum_{i=1}^{2} K_{i} B_{i}^{T}+A_{y}\right) y \\
& \leq c^{T} \alpha\left(\bar{A}_{2}\right) y .
\end{aligned}
$$

Then, $R_{0} \leq 1$ ensures that $d V_{\mathrm{DFE}}(x(t), y(t)) / d t \leq 0$ for all $y \geq$ 0 and that $d V_{\mathrm{DFE}}(x(t), y(t)) / d t=0$ holds when $(x(t), y(t))=$ $\left(x^{*}, 0\right)$. This proves the global asymptotic stability on $I R_{+}^{2 n+6}$ [14]. This achieves the proof that the DFE is globally and asymptotically stable.

\subsection{Existence of Endemic Equilibrium}

Definition 10. An equilibrium for a multipatch model as (1) is called endemic equilibrium when the two populations coexist (the density of each compartment is different from zero) at this equilibrium.

Lemma 11. An endemic equilibrium $(\bar{x}, \bar{y})$ of (1) can be determined, using an equation $F(\bar{x})=1$.

Proof. An endemic equilibrium $(\bar{x}, \bar{y})$ of (1) is obtained by setting the right-hand side of (4) which equals zero, giving

$$
\begin{aligned}
& \Lambda+D_{x} \bar{x}=\sum_{i=1}^{2}\left\langle\frac{B_{i}}{\bar{y}(t)}\right\rangle \frac{\left\langle e_{i}^{2} / \bar{x}(t)\right\rangle}{\bar{N}_{i}} e_{i}^{2}, \\
& \sum_{i=1}^{2}\left\langle\frac{B_{i}}{\bar{y}(t)}\right\rangle \frac{\left\langle e_{i}^{2} / \bar{x}(t)\right\rangle}{\bar{N}_{i}} K_{i}+A_{y} \bar{y}=0 .
\end{aligned}
$$

Multiplying the second equation of $(15)$ by $\left(-A_{y}\right)^{-1}$ gives

$$
\begin{aligned}
\bar{y}= & \left\langle\frac{B_{1}}{\bar{y}(t)}\right\rangle \frac{\left\langle e_{1}^{2} / \bar{x}(t)\right\rangle}{\bar{N}_{1}}\left(-A_{y}\right)^{-1} K_{1} \\
& +\left\langle\frac{B_{2}}{\bar{y}(t)}\right\rangle \frac{\left\langle e_{2}^{2} / \bar{x}(t)\right\rangle}{\bar{N}_{2}}\left(-A_{y}\right)^{-1} K_{2} .
\end{aligned}
$$

From (16),

$$
\begin{aligned}
& \left\langle\frac{B_{1}}{\bar{y}}\right\rangle=g_{11}\left\langle\frac{B_{1}}{\bar{y}}\right\rangle \frac{\left\langle e_{1}^{2} / \bar{x}(t)\right\rangle}{\bar{N}_{1}}+g_{12}\left\langle\frac{B_{2}}{\bar{y}}\right\rangle \frac{\left\langle e_{2}^{2} / \bar{x}(t)\right\rangle}{\bar{N}_{2}}, \\
& \left\langle\frac{B_{2}}{\bar{y}}\right\rangle=g_{21}\left\langle\frac{B_{1}}{\bar{y}}\right\rangle \frac{\left\langle e_{1}^{2} / \bar{x}(t)\right\rangle}{\bar{N}_{1}}+g_{22}\left\langle\frac{B_{2}}{\bar{y}}\right\rangle \frac{\left\langle e_{2}^{2} / \bar{x}(t)\right\rangle}{\bar{N}_{2}},
\end{aligned}
$$


where

$$
\begin{array}{ll}
g_{11}=\left\langle\frac{B_{1}}{\left(-A_{y}\right)^{-1} K_{1}}\right\rangle, & g_{12}=\left\langle\frac{B_{1}}{\left(-A_{y}\right)^{-1} K_{2}}\right\rangle, \\
g_{21}=\left\langle\frac{B_{2}}{\left(-A_{y}\right)^{-1} K_{1}}\right\rangle, & g_{22}=\left\langle\frac{B_{2}}{\left(-A_{y}\right)^{-1} K_{2}}\right\rangle .
\end{array}
$$

From the second equation of (17),

$$
\left\langle\frac{B_{2}}{\bar{y}}\right\rangle=\frac{g_{21}\left(\left\langle e_{1}^{2} / \bar{x}(t)\right\rangle / \bar{N}_{1}\right)}{1-g_{22}\left(\left\langle e_{2}^{2} / \bar{x}(t)\right\rangle / \bar{N}_{2}\right)}\left\langle\frac{B_{1}}{\bar{y}}\right\rangle \text {. }
$$

Using the expression of $\left\langle B_{2} / \bar{y}\right\rangle$ in (16) gives

$$
\begin{aligned}
\bar{y}= & \left\langle\frac{B_{1}}{\bar{y}}\right\rangle \frac{\left\langle e_{1}^{2} / \bar{x}(t)\right\rangle}{\bar{N}_{1}}\left(-A_{y}\right)^{-1} K_{1} \\
& +\frac{g_{21}\left(\left\langle e_{1}^{2} / \bar{x}(t)\right\rangle / \bar{N}_{1}\right)}{1-g_{22}\left(\left\langle e_{2}^{2} / \bar{x}(t)\right\rangle / \bar{N}_{2}\right)}\left\langle\frac{B_{1}}{\bar{y}}\right\rangle \\
& \times \frac{\left\langle e_{2}^{2} / \bar{x}(t)\right\rangle}{\bar{N}_{2}}\left(-A_{y}\right)^{-1} K_{2} .
\end{aligned}
$$

Then

$$
\begin{aligned}
\left\langle\frac{B_{1}}{\bar{y}}\right\rangle= & \left\langle\frac{B_{1}}{\bar{y}}\right\rangle \frac{\left\langle e_{1}^{2} / \bar{x}(t)\right\rangle}{\bar{N}_{1}} g_{11} \\
& +\frac{g_{21}\left(\left\langle e_{1}^{2} / \bar{x}(t)\right\rangle / \bar{N}_{1}\right)}{1-g_{22}\left(\left\langle e_{2}^{2} / \bar{x}(t)\right\rangle / \bar{N}_{2}\right)}\left\langle\frac{B_{1}}{\bar{y}}\right\rangle \frac{\left\langle e_{2}^{2} / \bar{x}(t)\right\rangle}{\bar{N}_{2}} g_{12},
\end{aligned}
$$

where $g_{11}=\left\langle B_{1} /\left(-A_{y}\right)^{-1} K_{1}\right\rangle$ and $g_{12}=\left\langle B_{1} /\left(-A_{y}\right)^{-1} K_{2}\right\rangle$.

If $\left\langle B_{1} / \bar{y}\right\rangle=0$, we obtain the disease-free equilibrium again. If not, the following equation holds:

$$
\begin{aligned}
1= & \frac{\left\langle e_{1}^{2} / \bar{x}(t)\right\rangle}{\bar{N}_{1}} \\
& \times\left[g_{11}+\frac{g_{21} g_{12}}{1-g_{22}\left(\left\langle e_{2}^{2} / \bar{x}(t)\right\rangle / \bar{N}_{2}\right)} \frac{\left\langle e_{2}^{2} / \bar{x}(t)\right\rangle}{\bar{N}_{2}}\right],
\end{aligned}
$$

which can also be written as

$$
\left\langle\frac{e_{2}^{2}}{\bar{x}(t)}\right\rangle=\frac{\bar{N}_{1} \bar{N}_{2}-g_{11} \bar{N}_{2}\left\langle e_{1}^{2} / \bar{x}(t)\right\rangle}{g_{12} g_{21}\left\langle e_{1}^{2} / \bar{x}(t)\right\rangle+g_{22} \bar{N}_{1}-g_{11} g_{22}\left\langle e_{1}^{2} / \bar{x}(t)\right\rangle} .
$$

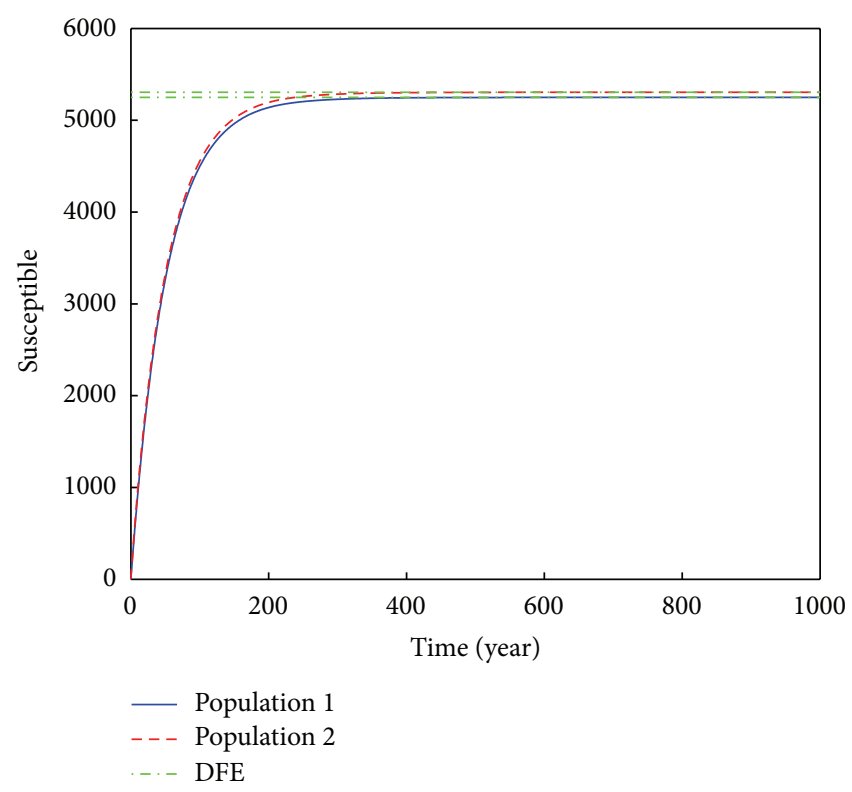

FIGURE 2: Trajectories of susceptible individuals of (1) for different initial conditions when $\beta_{1}=0.002$ and $\beta_{2}=0.003$ (such that $R_{0}^{1}=$ 0.3691 and $R_{0}^{2}=0.4980$ ). We can observe the global stability of DFE when $R_{0}<1$. All other parameters are as in Table 1.

This expression of $\left\langle e_{2}^{2} / \bar{x}(t)\right\rangle$ in (23) is positive when one of these conditions is satisfied:

$$
\begin{gathered}
\bar{N}_{1}-g_{11}\left\langle\frac{e_{1}^{2}}{\bar{x}(t)}\right\rangle>0, \\
\bar{N}_{1}-g_{11}\left\langle\frac{e_{1}^{2}}{\bar{x}(t)}\right\rangle<0, \\
g_{12} g_{21}\left\langle\frac{e_{1}^{2}}{\bar{x}(t)}\right\rangle+g_{22} \bar{N}_{1}<g_{11} g_{22}\left\langle\frac{e_{1}^{2}}{\bar{x}(t)}\right\rangle .
\end{gathered}
$$

Case 1. $\bar{N}_{1}-g_{11}\left\langle e_{1}^{2} / \bar{x}(t)\right\rangle>0$. This yields the condition

$$
\left\langle\frac{e_{1}^{2}}{\bar{x}(t)}\right\rangle<\frac{\bar{N}_{1}}{g_{11}}
$$

Case 2. $\bar{N}_{1}-g_{11}\left\langle e_{1}^{2} / \bar{x}(t)\right\rangle<0$ and $g_{12} g_{21}\left\langle e_{1}^{2} / \bar{x}(t)\right\rangle+g_{22} \bar{N}_{1}<$ $g_{11} g_{22}\left\langle e_{1}^{2} / \bar{x}(t)\right\rangle$. This yields the condition

$$
\begin{gathered}
\left\langle\frac{e_{1}^{2}}{\bar{x}(t)}\right\rangle>\max \left(\frac{\bar{N}_{1}}{g_{11}}, \frac{g_{22} \bar{N}_{1}}{g_{11} g_{22}-g_{12} g_{21}}\right) \\
\text { since } g_{11} g_{22}-g_{12} g_{21}>0 .
\end{gathered}
$$

In sum, $\left\langle e_{2}^{2} / \bar{x}(t)\right\rangle$ exists when $\left\langle e_{1}^{2} / \bar{x}(t)\right\rangle$ satisfies condition (25) or condition (26). Now, using the first equation of system (15) gives

$$
\Lambda+D_{x} \bar{x}=\sum_{i=1}^{2}\left\langle\frac{B_{i}}{\bar{y}(t)}\right\rangle \frac{\left\langle e_{i}^{2} / \bar{x}(t)\right\rangle}{\bar{N}_{i}} e_{i}^{2} .
$$



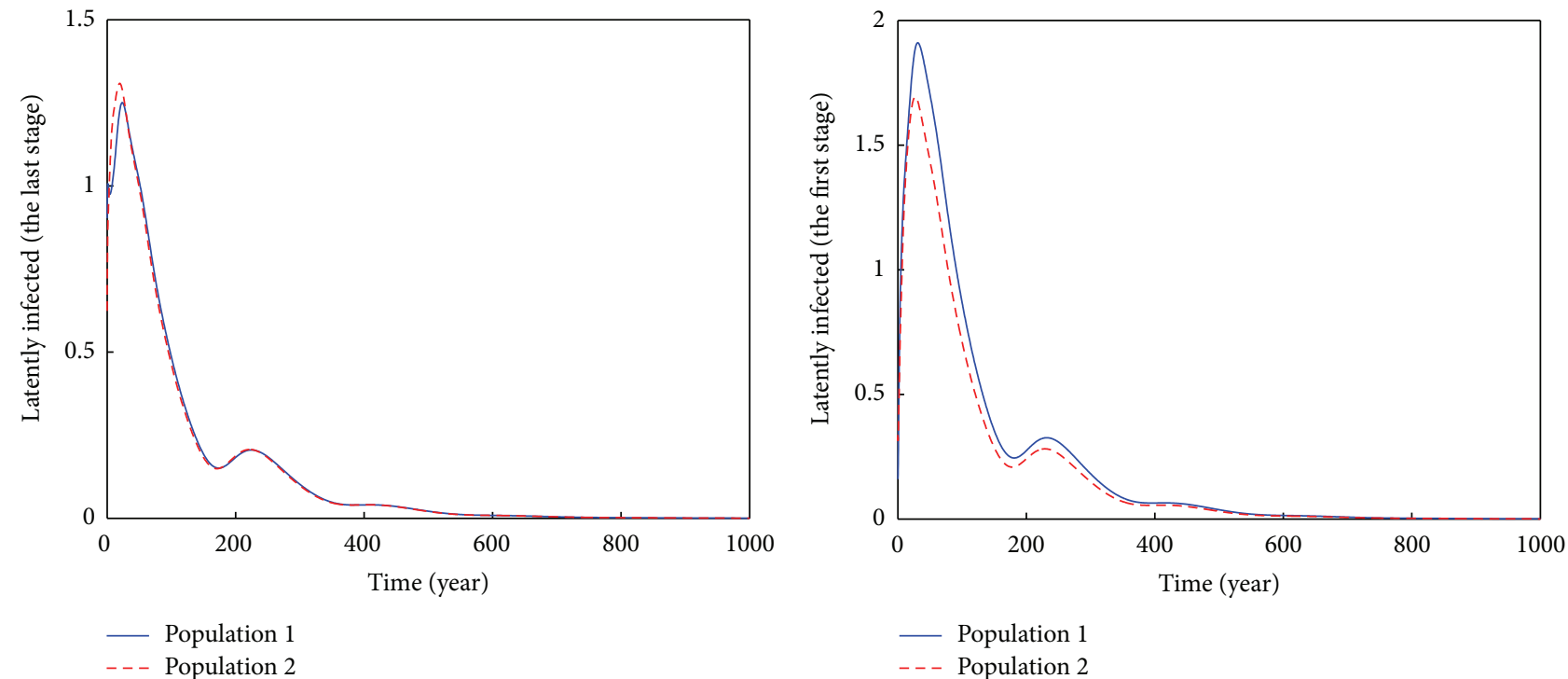

FIGURE 3: Trajectories of latently infected individuals of (1) for different initial conditions when $\beta_{1}=0.002$ and $\beta_{2}=0.003$ (such that $R_{0}^{1}=0.3691$ and $\left.R_{0}^{2}=0.4980\right)$. The latent classes disappear at the end and only susceptible classes persist when $R_{0}<1$. All other parameters are as in Table 1.

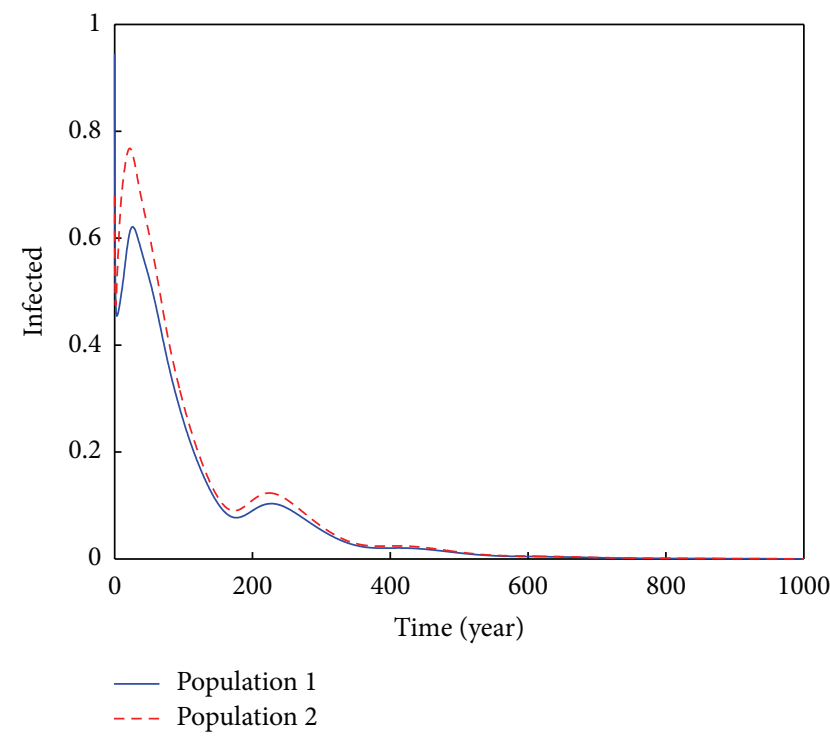

(a)

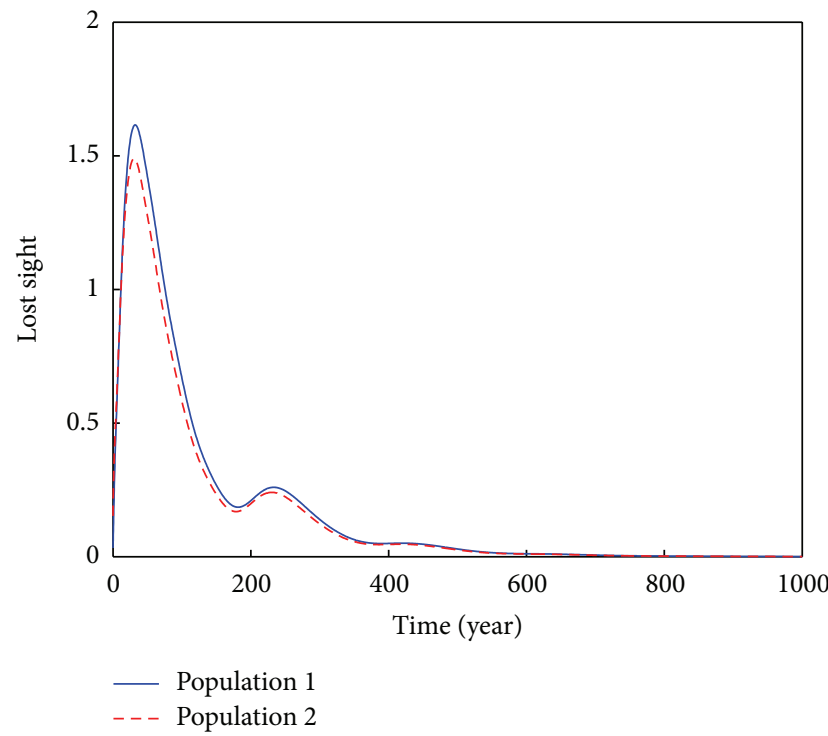

(b)

FIGURE 4: Trajectories of infectious individuals in care center (a) and lost sight individuals (b) of (1) for different initial conditions when $\beta_{1}=0.002$ and $\beta_{2}=0.003$ (such that $R_{0}^{1}=0.3691$ and $R_{0}^{2}=0.4980$ ). The infectious and lost sight classes disappear at the end and only susceptible classes persist when $R_{0}<1$. All other parameters are as in Table 1 .

Then,

$$
\begin{aligned}
& \frac{\left\langle B_{1} / \bar{y}\right\rangle}{\bar{N}_{1}}=\frac{\Lambda_{1}-\left(\mu_{1}+a_{1}\right) \bar{x}_{1}+a_{2} \bar{x}_{2}}{\bar{x}_{1}}=\frac{\left\langle\Lambda+D_{x} \bar{x} / e_{1}^{2}\right\rangle}{\left\langle e_{1}^{2} / \bar{x}\right\rangle}, \\
& \frac{\left\langle B_{2} / \bar{y}\right\rangle}{\bar{N}_{2}}=\frac{\Lambda_{2}-\left(\mu_{2}+a_{2}\right) \bar{x}_{2}+a_{1} \bar{x}_{1}}{\bar{x}_{2}}=\frac{\left\langle\Lambda+D_{x} \bar{x} / e_{2}^{2}\right\rangle}{\left\langle e_{2}^{2} / \bar{x}\right\rangle} .
\end{aligned}
$$

Putting (28) into the first equation of (17) gives

$$
\begin{aligned}
\bar{N}_{1}= & \left\langle\frac{e_{1}^{2}}{\bar{x}(t)}\right\rangle \\
& \times\left[g_{11}\left\langle\Lambda+\frac{D_{x} \bar{x}}{e_{1}^{2}}\right\rangle+g_{12}\left\langle\Lambda+\frac{D_{x} \bar{x}}{e_{2}^{2}}\right\rangle\right] \\
& \times\left(\left\langle\Lambda+\frac{D_{x} \bar{x}}{e_{1}^{2}}\right\rangle\right)^{-1} .
\end{aligned}
$$




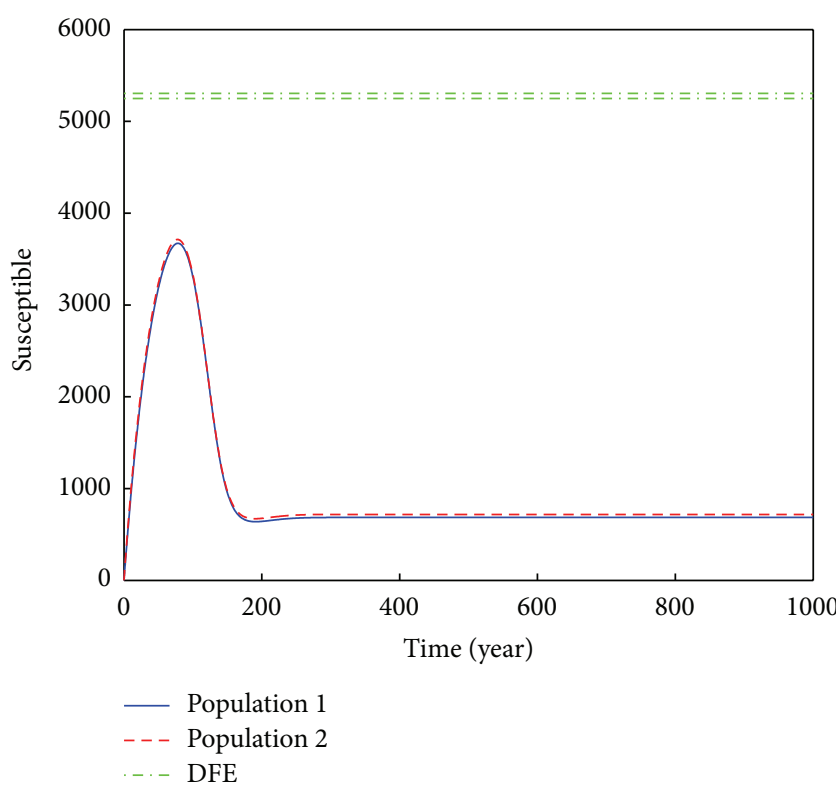

Figure 5: Trajectories of susceptible individuals of (1) for different initial conditions when $\beta_{1}=0.002$ and $\beta_{2}=0.003$ (such that $R_{0}^{1}=0.3691$ and $R_{0}^{2}=0.4980$ ). The DFE coexists with endemic equilibrium and is locally stable when $R_{0}<1$. All other parameters are as in Table 1.

Putting (28) into the second equation of (17) gives

$$
\begin{aligned}
\bar{N}_{2}= & \left\langle\frac{e_{2}^{2}}{\bar{x}(t)}\right\rangle \\
& \times\left[g_{21}\left\langle\Lambda+\frac{D_{x} \bar{x}}{e_{1}^{2}}\right\rangle+g_{22}\left\langle\Lambda+\frac{D_{x} \bar{x}}{e_{2}^{2}}\right\rangle\right] \\
& \times\left(\left\langle\Lambda+\frac{D_{x} \bar{x}}{e_{2}^{2}}\right\rangle\right)^{-1} .
\end{aligned}
$$

Then, in the compact form,

$$
\begin{gathered}
\frac{\bar{N}_{1}}{\left\langle e_{1}^{2} / \bar{x}(t)\right\rangle}=\frac{g_{11}\left\langle\Lambda+D_{x} \bar{x} / e_{1}^{2}\right\rangle+g_{12}\left\langle\Lambda+D_{x} \bar{x} / e_{2}^{2}\right\rangle}{\left\langle\Lambda+D_{x} \bar{x} / e_{1}^{2}\right\rangle}, \\
\frac{\bar{N}_{2}}{\left\langle e_{2}^{2} / \bar{x}(t)\right\rangle}=\frac{g_{21}\left\langle\Lambda+D_{x} \bar{x} / e_{1}^{2}\right\rangle+g_{22}\left\langle\Lambda+D_{x} \bar{x} / e_{2}^{2}\right\rangle}{\left\langle\Lambda+D_{x} \bar{x} / e_{2}^{2}\right\rangle} .
\end{gathered}
$$

From (1),

$$
\begin{aligned}
\dot{N}_{1} & +\dot{N}_{2} \\
& =\Lambda_{1}+\Lambda_{2}-\mu_{1} N_{1}-\mu_{2} N_{2}-d_{1} I_{1}-l_{1} L_{1}-d_{2} I_{2}-l_{2} L_{2},
\end{aligned}
$$

which can be written in semicompact form as

$$
\dot{N}_{1}+\dot{N}_{2}=\Lambda_{1}+\Lambda_{2}-\mu_{1} N_{1}-\mu_{2} N_{2}-\left\langle\frac{B_{3}}{y}\right\rangle-\left\langle\frac{B_{4}}{y}\right\rangle \text {, }
$$

where $B_{3}=\left(0, \ldots, 0, d_{1}, l_{1}, 0, \ldots, 0,0,0\right)^{T}, B_{4}=(0, \ldots, 0,0$, $\left.0,0, \ldots, 0, d_{2}, l_{2}\right)^{T}$, and $N=N_{1}+N_{2}$.

At equilibrium,

$$
\Lambda_{1}+\Lambda_{2}=\mu_{1} \bar{N}_{1}+\mu_{2} \bar{N}_{2}+\left\langle\frac{B_{3}}{\bar{y}}\right\rangle+\left\langle\frac{B_{4}}{\bar{y}}\right\rangle .
$$

Using (16) in (34),

$$
\begin{aligned}
\Lambda_{1}+\Lambda_{2}= & \mu_{1} \bar{N}_{1}+\mu_{2} \bar{N}_{2}+\left\langle\frac{B_{1}}{\bar{y}(t)}\right\rangle \frac{\left\langle e_{1}^{2} / \bar{x}(t)\right\rangle}{\bar{N}_{1}} h_{31} \\
& +\left\langle\frac{B_{2}}{\bar{y}(t)}\right\rangle \frac{\left\langle e_{2}^{2} / \bar{x}(t)\right\rangle}{\bar{N}_{2}} h_{32} \\
& +\left\langle\frac{B_{1}}{\bar{y}(t)}\right\rangle \frac{\left\langle e_{1}^{2} / \bar{x}(t)\right\rangle}{\bar{N}_{1}} h_{41} \\
& +\left\langle\frac{B_{2}}{\bar{y}(t)}\right\rangle \frac{\left\langle e_{2}^{2} / \bar{x}(t)\right\rangle}{\bar{N}_{2}} h_{42},
\end{aligned}
$$

where $h_{31}=\left\langle B_{3} /\left(-A_{y}\right)^{-1} K_{1}\right\rangle, h_{32}=\left\langle B_{3} /\left(-A_{y}\right)^{-1} K_{2}\right\rangle, h_{41}=$ $\left\langle B_{4} /\left(-A_{y}\right)^{-1} K_{1}\right\rangle$, and $h_{42}=\left\langle B_{4} /\left(-A_{y}\right)^{-1} K_{2}\right\rangle$. Using (28) in (35) gives

$$
\begin{aligned}
\Lambda_{1}+\Lambda_{2}= & \mu_{1} \bar{N}_{1}+\mu_{2} \bar{N}_{2}+\left\langle\Lambda+\frac{D_{x} \bar{x}}{e_{1}^{2}}\right\rangle h_{31} \\
& +\left\langle\Lambda+\frac{D_{x} \bar{x}}{e_{2}^{2}}\right\rangle h_{32} \\
& +\left\langle\Lambda+\frac{D_{x} \bar{x}}{e_{1}^{2}}\right\rangle h_{41}+\left\langle\Lambda+\frac{D_{x} \bar{x}}{e_{2}^{2}}\right\rangle h_{42}
\end{aligned}
$$

Using (31) in (36) gives

$$
\begin{aligned}
1= & \frac{\mu_{1}}{\Lambda_{1}+\Lambda_{2}}\left\langle\frac{e_{1}^{2}}{\bar{x}}\right\rangle \\
& \times \frac{g_{11}\left\langle\Lambda+D_{x} \bar{x} / e_{1}^{2}\right\rangle+g_{12}\left\langle\Lambda+D_{x} \bar{x} / e_{2}^{2}\right\rangle}{\left\langle\Lambda+D_{x} \bar{x} / e_{1}^{2}\right\rangle} \\
& +\frac{\mu_{2}}{\Lambda_{1}+\Lambda_{2}}\left\langle\frac{e_{2}^{2}}{\bar{x}}\right\rangle \\
& \times \frac{g_{21}\left\langle\Lambda+D_{x} \bar{x} / e_{1}^{2}\right\rangle+g_{22}\left\langle\Lambda+D_{x} \bar{x} / e_{2}^{2}\right\rangle}{\left\langle\Lambda+D_{x} \bar{x} / e_{2}^{2}\right\rangle} \\
& +\frac{h_{31}}{\Lambda_{1}+\Lambda_{2}}\left\langle\Lambda+\frac{D_{x} \bar{x}}{e_{1}^{2}}\right\rangle+\frac{h_{32}}{\Lambda_{1}+\Lambda_{2}}\left\langle\Lambda+\frac{D_{x} \bar{x}}{e_{2}^{2}}\right\rangle \\
& +\frac{h_{41}}{\Lambda_{1}+\Lambda_{2}}\left\langle\Lambda+\frac{D_{x} \bar{x}}{e_{1}^{2}}\right\rangle+\frac{h_{42}}{\Lambda_{1}+\Lambda_{2}}\left\langle\Lambda+\frac{D_{x} \bar{x}}{e_{2}^{2}}\right\rangle .
\end{aligned}
$$



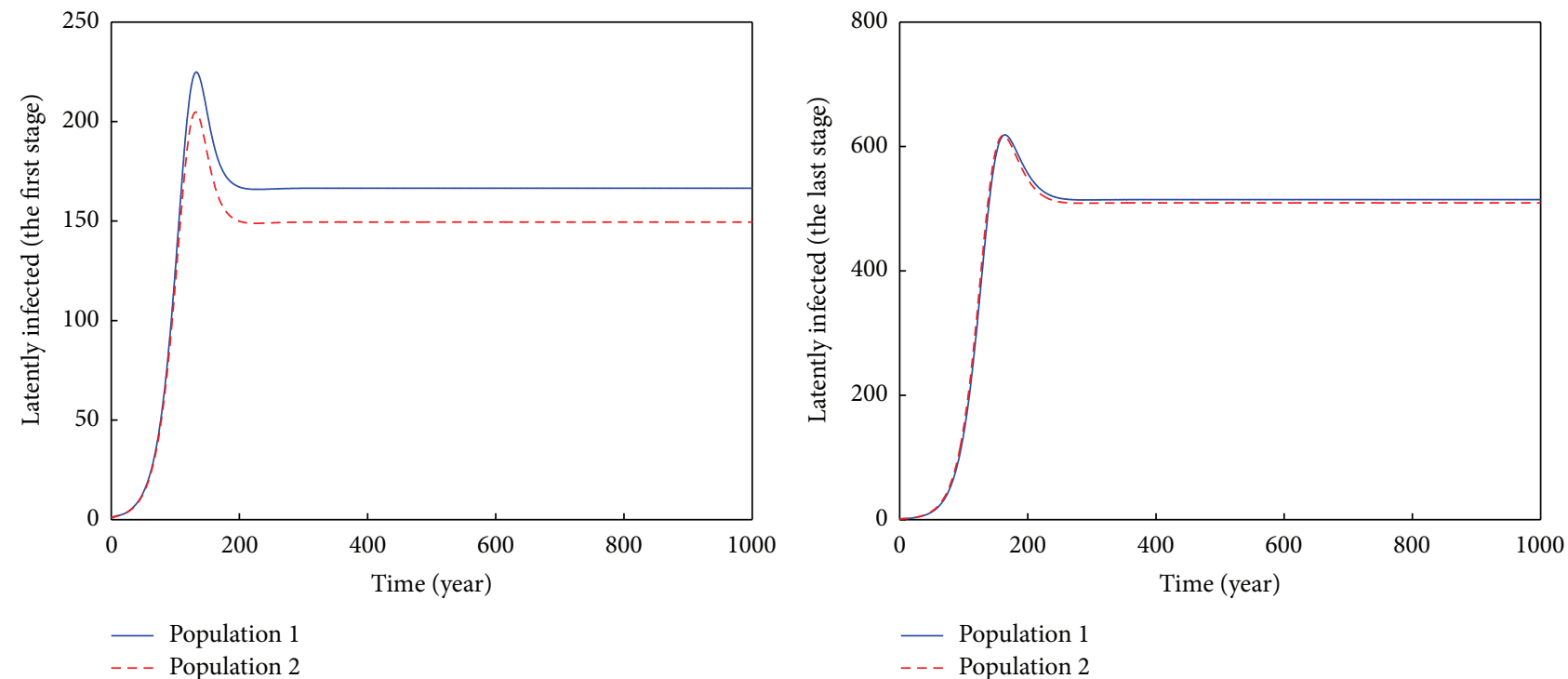

FIGURE 6: Trajectories of latently infected individuals of (1) for different initial conditions when $\beta_{1}=0.822$ and $\beta_{2}=0.943$ (such that $R_{0}^{1}=2.3601$ and $\left.R_{0}^{2}=2.4910\right)$. The endemic equilibrium is locally and asymptotically stable when $R_{0}>1$. All other parameters are as in Table 1.
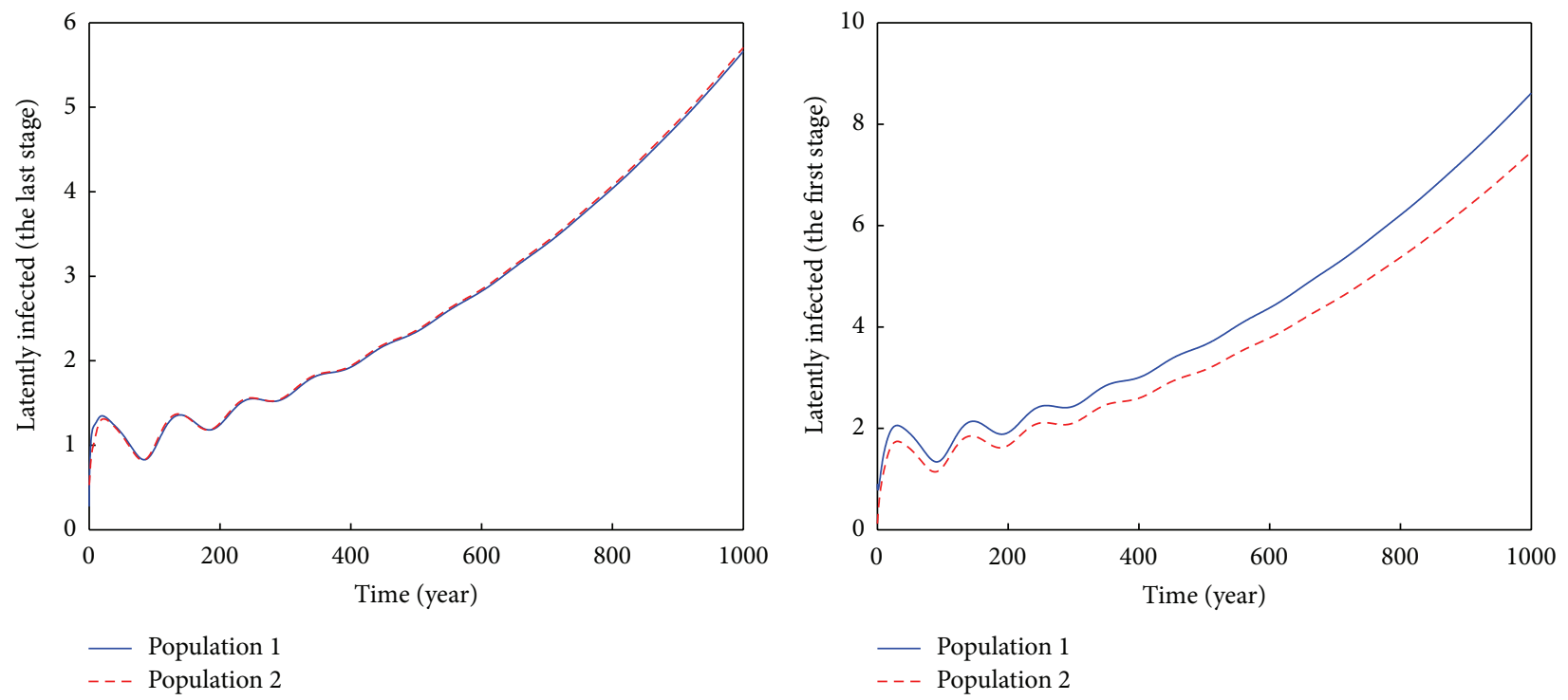

FIGURE 7: Trajectories of latently infected individuals of (1) for different initial conditions when $\beta_{1}=2.082$ and $\beta_{2}=2.173$ (such that $R_{0}^{1}=3.1492$ and $R_{0}^{2}=4.1085$ ). The endemic equilibrium is locally and asymptotically stable when $R_{0}>1$. In this case, the DFE is unstable. All other parameters are as in Table 1.

Let us set

$$
\begin{aligned}
F(\bar{x})= & \frac{\mu_{1}}{\Lambda_{1}+\Lambda_{2}}\left\langle\frac{e_{1}^{2}}{\bar{x}}\right\rangle \\
& \times \frac{g_{11}\left\langle\Lambda+D_{x} \bar{x} / e_{1}^{2}\right\rangle+g_{12}\left\langle\Lambda+D_{x} \bar{x} / e_{2}^{2}\right\rangle}{\left\langle\Lambda+D_{x} \bar{x} / e_{1}^{2}\right\rangle} \\
& +\frac{\mu_{2}}{\Lambda_{1}+\Lambda_{2}}\left\langle\frac{e_{2}^{2}}{\bar{x}}\right\rangle \\
& \times \frac{g_{21}\left\langle\Lambda+D_{x} \bar{x} / e_{1}^{2}\right\rangle+g_{22}\left\langle\Lambda+D_{x} \bar{x} / e_{2}^{2}\right\rangle}{\left\langle\Lambda+D_{x} \bar{x} / e_{2}^{2}\right\rangle}
\end{aligned}
$$




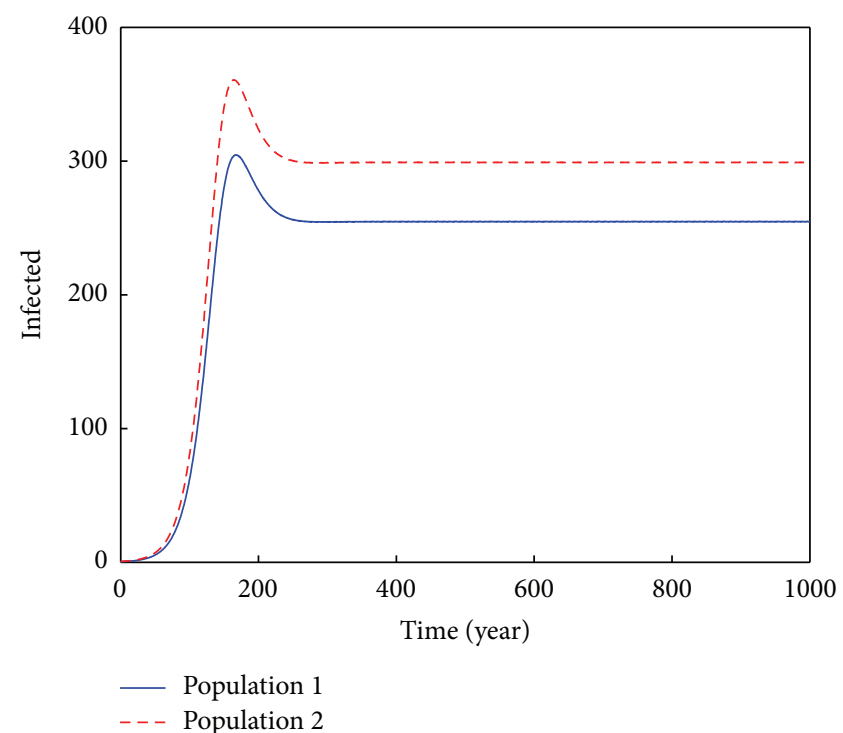

(a)

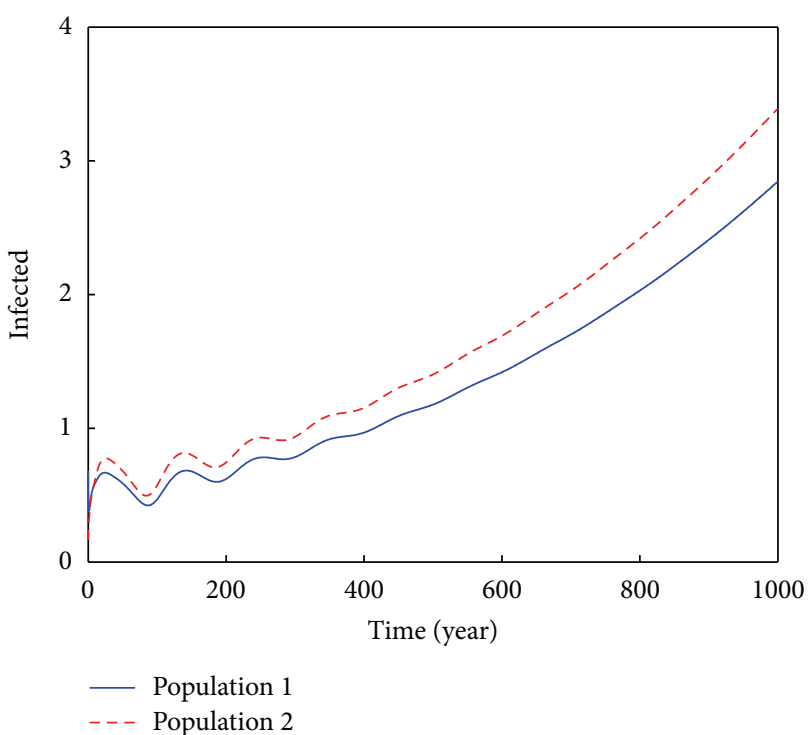

(b)

Figure 8: ( $(a, b)$ Trajectories of infectious individuals in care center of (1) for different initial conditions. The endemic equilibrium exists and is stable when $R_{0}>1$; the DFE is unstable in this case.

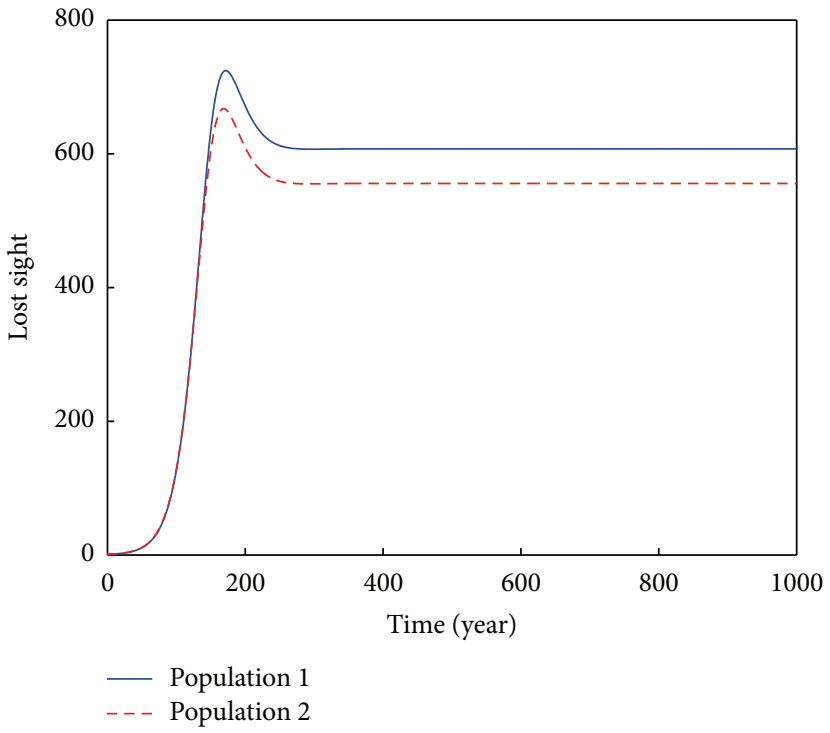

(a)

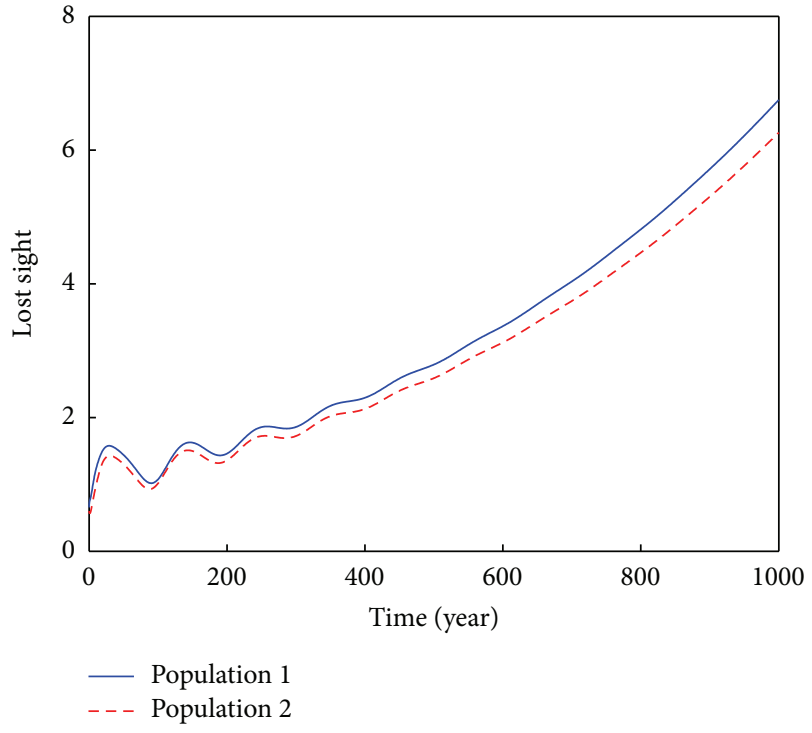

(b)

FIGURE 9: (a, b) Trajectories of lost sight individuals of (1) for different initial conditions. The endemic equilibrium exists and is stable when $R_{0}>1$; the DFE is unstable in this case.

One can observe that it is useful to analyze how many times the function $F(\bar{x})$ intersects the line $F(\bar{x})=1$ in the plane $(\bar{x}, F(\bar{x}))$. The monotony of the function $F$ depends on the parameters of system (1). Then, the following lemma and theorem hold.

Lemma 12. The function F satisfies the following properties.
(1) The limit when $\bar{x} \rightarrow 0$ is finite and is given by

$$
\begin{aligned}
\lim _{\bar{x} \rightarrow 0} F(\bar{x})= & \frac{h_{31}}{\Lambda_{1}+\Lambda_{2}}\left\langle\frac{\Lambda}{e_{1}^{2}}\right\rangle+\frac{h_{32}}{\Lambda_{1}+\Lambda_{2}}\left\langle\frac{\Lambda}{e_{2}^{2}}\right\rangle \\
& +\frac{h_{41}}{\Lambda_{1}+\Lambda_{2}}\left\langle\frac{\Lambda}{e_{1}^{2}}\right\rangle+\frac{h_{42}}{\Lambda_{1}+\Lambda_{2}}\left\langle\frac{\Lambda}{e_{2}^{2}}\right\rangle=s_{0} .
\end{aligned}
$$


TABLE 1: Numerical values for the parameters of the model.

\begin{tabular}{|c|c|c|c|}
\hline Parameters & Description & Estimated value/range & Reference \\
\hline$\Lambda_{1}$ & Recruitment rate into the $S_{1}$ class & $100 / \mathrm{yr}$ & Assumed \\
\hline$\Lambda_{2}$ & Recruitment rate into the $S_{2}$ class & $110 / y r$ & Assumed \\
\hline$\beta_{1}$ & Transmission coefficient in patch 1 & Variable & Assumed \\
\hline$\beta_{2}$ & Transmission coefficient in patch 2 & Variable & Assumed \\
\hline$\mu_{1}$ & Per capita death rate in patch 1 & $0.019896 / \mathrm{yr}$ & Estimated \\
\hline$\mu_{2}$ & Per capita death rate in patch 2 & $0.019897 / \mathrm{yr}$ & Estimated \\
\hline$k_{1 i}$ & Progression rate from $E_{1 i}$ to next class in patch 1 & $0.00013 / \mathrm{yr}$ & Assumed \\
\hline$k_{2 i}$ & Progression rate from $E_{2 i}$ to next class in patch 2 & $0.00023 / \mathrm{yr}$ & Assumed \\
\hline$r_{1 i}$ & Effective chemoprophylaxis in the $E_{1 i}$ class & $0 / \mathrm{yr}$ & Estimated \\
\hline$r_{2 i}$ & Effective chemoprophylaxis in the $E_{2 i}$ class & $0 / \mathrm{yr}$ & Estimated \\
\hline$\delta_{1}$ & Lost sight individuals still able to transmit disease in patch 1 & $0.46 / \mathrm{yr}$ & Estimated \\
\hline$\delta_{2}$ & Lost sight individuals still able to transmit disease in patch 2 & $0.48 / \mathrm{yr}$ & Estimated \\
\hline$\gamma_{1}$ & Effective therapy in $I_{1}$ class & $0.8182 / \mathrm{yr}$ & Estimated \\
\hline$\gamma_{2}$ & Effective therapy in $I_{2}$ class & $0.8183 / \mathrm{yr}$ & Assumed \\
\hline$\alpha_{1}$ & Return rate in care centre for patch 1 & $0.23 / \mathrm{yr}$ & Estimated \\
\hline$\alpha_{2}$ & Return rate in care centre for patch 2 & $0.23 / \mathrm{yr}$ & Estimated \\
\hline$v_{1}$ & Infectious individuals in care centre of patch 1 who can stay in care centre & $0.59 / \mathrm{yr}$ & Estimated \\
\hline$v_{2}$ & Infectious individuals in care centre of patch 2 who can stay in care centre & $0.5903 / \mathrm{yr}$ & Estimated \\
\hline$s_{1}$ & Infectious individuals in care centre of patch 1 who disappear & $0.019 / \mathrm{yr}$ & Estimated \\
\hline$s_{2}$ & Infectious individuals in care centre of patch 1 who disappear & $0.01905 / \mathrm{yr}$ & Estimated \\
\hline$a_{1}$ & Migration rate of susceptible individuals from patch 1 to patch 2 & $0.07 / \mathrm{yr}$ & Assumed \\
\hline$a_{2}$ & Migration rate of susceptible individuals from patch 2 to patch 1 & $0.0701 / \mathrm{yr}$ & Assumed \\
\hline$b_{1 i}$ & Migration rate from latent class $E_{1 i}$ to latent class $E_{2 i}$ & $0.05 / \mathrm{yr}$ & Assumed \\
\hline$b_{2 i}$ & Migration rate from latent class $E_{2 i}$ to latent class $E_{1 i}$ & $0.0503 / \mathrm{yr}$ & Assumed \\
\hline$c_{1}$ & Migration rate of infectious individuals from patch 1 to patch 2 & $0.02 / \mathrm{yr}$ & Assumed \\
\hline$c_{2}$ & Migration rate of infectious individuals from patch 2 to patch 1 & $0.0201 / \mathrm{yr}$ & Assumed \\
\hline$d_{1}$ & Additional death rate in $I_{1}$ & $0.0575 / \mathrm{yr}$ & Assumed \\
\hline$d_{2}$ & Additional death rate in $I_{2}$ & $0.05751 / \mathrm{yr}$ & Assumed \\
\hline$l_{1}$ & Additional death rate in $L_{1}$ & $0.0565 / \mathrm{yr}$ & Assumed \\
\hline$l_{2}$ & Additional death rate in $L_{2}$ & $0.05651 / \mathrm{yr}$ & Assumed \\
\hline$p_{1}$ & Fast progression to the $I_{1}$ class & $0.015 / \mathrm{yr}$ & Assumed \\
\hline$p_{2}$ & Fast progression to the $I_{2}$ class & 0.016 & Assumed \\
\hline
\end{tabular}

(2) The limit when $\bar{x} \rightarrow+\infty$ is infinite: $\lim _{\bar{x} \rightarrow+\infty} F(\bar{x})=$ $+\infty$.

Theorem 13. These properties hold for system (1).

(1) There is no boundary equilibrium. This means that the disease cannot persist in one patch while disappearing in the other one.

(2) If $s_{0} \leq R_{0} \leq 1$, there exists at least one solution of $F(\bar{x})=1$, depending on the monotony of the function $F$, and therefore there exists at least one endemic equilibrium $(\bar{x}, \bar{y})$ for system (5) which coexists with the disease-free equilibrium. This situation corresponds to a backward bifurcation.

(3) If $R_{0} \leq s_{0} \leq 1$, there exists at least one solution of $F(\bar{x})=1$, depending on the monotony of the function $F$, and therefore there exists at least one endemic equilibrium $(\bar{x}, \bar{y})$ for system (5) which coexists with the disease-free equilibrium. There is no backward bifurcation in this case since the endemic equilibrium no longer exists (it disappears as the disease-free equilibrium).

(4) If $R_{0} \leq 1 \leq s_{0}$, at least one solution of $F(\bar{x})=1$ can exist, depending on parameters of system (1), since the monotony of the function $F$ also depends on these parameters.

(5) If $s_{0} \leq 1 \leq R_{0}$, at least one solution of $F(\bar{x})=1$ can exist, depending on the monotony of the function $F$, and therefore there exists at least one endemic equilibrium $(\bar{x}, \bar{y})$ for system (5), which coexists with the diseasefree equilibrium.

(6) If $1 \leq R_{0} \leq s_{0}$, at least one solution of $F(\bar{x})=1$ can exist, depending on parameters of system (1), since the monotony of the function $F$ also depends on these parameters.

(7) If $1 \leq s_{0} \leq R_{0}$, at least one solution of $F(\bar{x})=1$ can exist, depending on parameters of system (1), since 
the monotony of the function $F$ also depends on these parameters.

Proof. When an endemic equilibrium $(\bar{x}, \bar{y})$ for system (5) exists, $\bar{x}$ is solution of $F(\bar{x})=1 . \bar{N}_{1}$ and $\bar{N}_{2}$ are given by (31) as

$$
\begin{aligned}
& \frac{\bar{N}_{1}}{\left\langle e_{1}^{2} / \bar{x}(t)\right\rangle}=\frac{g_{11}\left\langle\Lambda+D_{x} \bar{x} / e_{1}^{2}\right\rangle+g_{12}\left\langle\Lambda+D_{x} \bar{x} / e_{2}^{2}\right\rangle}{\left\langle\Lambda+D_{x} \bar{x} / e_{1}^{2}\right\rangle}, \\
& \frac{\bar{N}_{2}}{\left\langle e_{2}^{2} / \bar{x}(t)\right\rangle}=\frac{g_{21}\left\langle\Lambda+D_{x} \bar{x} / e_{1}^{2}\right\rangle+g_{22}\left\langle\Lambda+D_{x} \bar{x} / e_{2}^{2}\right\rangle}{\left\langle\Lambda+D_{x} \bar{x} / e_{2}^{2}\right\rangle} .
\end{aligned}
$$

$\left\langle B_{1} / \bar{y}\right\rangle$ and $\left\langle B_{2} / \bar{y}\right\rangle$ are given by (16) as $\left\langle B_{i} / \bar{y}\right\rangle / \bar{N}_{i}=\langle\Lambda+$ $\left.D_{x} \bar{x} / e_{i}^{2}\right\rangle /\left\langle e_{i}^{2} / \bar{x}\right\rangle, i=1,2$.

The expression of $\bar{y}$ is given by (16) as

$$
\begin{aligned}
\bar{y}= & \left\langle\frac{B_{1}}{\bar{y}}\right\rangle \frac{\left\langle e_{1}^{2} / \bar{x}(t)\right\rangle}{\bar{N}_{1}}\left(-A_{y}\right)^{-1} K_{1} \\
& +\left\langle\frac{B_{2}}{\bar{y}}\right\rangle \frac{\left\langle e_{2}^{2} / \bar{x}(t)\right\rangle}{\bar{N}_{2}}\left(-A_{y}\right)^{-1} K_{2} .
\end{aligned}
$$

Since the function $F$ is not strictly monotone, the number of solutions of $F(\bar{x})=1$ depends on the parameters of system (1). Therefore, there can be more than one endemic equilibrium.

Remark 14. In the second case of the theorem, we observe that at least one endemic equilibrium coexists with the disease-free equilibrium. Then, there can be a backward bifurcation in this case.

3.6. Stability of Endemic Equilibrium. The stability of endemic equilibrium is always a big challenge in epidemiology. The problem is more difficult here since we have $2 n+6$ equations. For multipatch and universal incidence law, results concerning the global stability of endemic equilibrium are limited. Next we will illustrate some results concerning our model.

\section{Numerical Simulations}

System (1) is simulated with parameter values presented in Table 1 using real data of the cities of Yaounde and Bafia [21].

Next we use the values in Table 1 to generate several curves (Figures 2, 3, 4, 5, 6, 7, 8, and 9) in order to illustrate our theoretical results.

\section{Conclusion}

The long-term dynamics of our system has been completely investigated. The model exhibits rich dynamics, depending on the values of the bifurcation parameters $R_{0}^{i}, i=1,2$, and $R_{0}$. The influence of parameter $R_{0}^{i}$ is significant on the spread of tuberculosis since it quantifies the intensity of pathogens transmission. The stability of equilibria depends on these parameters. We have transcritical bifurcation parameters and backward bifurcation. When the basic reproduction number is less than unity, tuberculosis can be controlled in each population if the DFE is the unique equilibrium. It can be more difficult if the DFE coexists with at least one endemic equilibrium (this is the situation of backward bifurcation). In this case, the disease-free equilibrium can be locally and asymptotically stable, as well as the endemic equilibrium. When the basic reproduction number $R_{0}$ is greater than unity, tuberculosis is endemic and can be difficult to control in the population. The disease in our model cannot persist in one population while disappearing in the other one.

\section{Disclosure}

Part of this work was realized during the visit of Jean Jules Tewa at the UMI 209 UMMISCO Laboratory of University Cheikh Anta Diop, Dakar, Senegal.

\section{Conflict of Interests}

The authors declare that there is no conflict of interests regarding the publication of this paper.

\section{References}

[1] J. K. Hale, Asymptotic Behavior of Dissipative Systems, American Mathematical Society, Providence, RI, USA, 1988.

[2] I. Bendixson, "Sur les courbes définies par des équations différentielles," Acta Mathematica, vol. 24, no. 1, pp. 1-88, 1901.

[3] F. Verhulst, Nonlinear Differential Equations and Dynamical Systems, Universitext, Springer, Berlin, Germany, 1990.

[4] H. Dulac, "Recherche des cycles limites," Comptes Rendus de l'Académie des Sciences, vol. 204, pp. 1703-1706, 1937.

[5] M. Y. Li and J. S. Muldowney, "On R. A. Smith's autonomous convergence theorem," The Rocky Mountain Journal of Mathematics, vol. 25, no. 1, pp. 365-379, 1995.

[6] M. Y. Li and J. S. Muldowney, "A geometric approach to globalstability problems," SIAM Journal on Mathematical Analysis, vol. 27, no. 4, pp. 1070-1083, 1996.

[7] M. Y. Li, J. S. Muldowney, and P. van den Driessche, "Global stability of SEIRS models in epidemiology," The Canadian Applied Mathematics Quarterly, vol. 7, no. 4, pp. 409-425, 1999.

[8] M. Y. Li, H. L. Smith, and L. Wang, "Global dynamics an SEIR epidemic model with vertical transmission," SIAM Journal on Applied Mathematics, vol. 62, no. 1, pp. 58-69, 2001.

[9] C. C. McCluskey and P. van den Driessche, "Global analysis of two tuberculosis models," Journal of Dynamics and Differential Equations, vol. 16, no. 1, pp. 139-166, 2004.

[10] M. M. Ballyk, C. C. McCluskey, and G. S. K. Wolkowicz, "Global analysis of competition for perfectly substitutable resources with linear response," Journal of Mathematical Biology, vol. 51, no. 4, pp. 458-490, 2005.

[11] A. B. Gumel, C. C. McCluskey, and J. Watmough, "Modelling the potential impact of a SARS vaccine," Mathematical Biosciences and Engineering, vol. 3, no. 3, pp. 485-512, 2006.

[12] J. J. Tewa, S. Bowong, B. Mewoli, and J. Kurths, "Two-patch transmission of tuberculosis," Mathematical Population Studies, vol. 18, no. 3, pp. 189-205, 2011. 
[13] J. P. LaSalle, The Stability of Dynamical Systems, SIAM, Philadelphia, Pa, USA, 1976.

[14] A. Lyapunov, Problème Général de la Stabilité du Mouvement, vol. 17 of Annals of Mathematics Studies, Princeton University Press, Princeton, NJ, USA, 1949.

[15] A. Iggidr, J. Kamgang, G. Sallet, and J. Tewa, "Global analysis of new malaria intrahost models with a competitive exclusion principle," SIAM Journal on Applied Mathematics, vol. 67, no. 1, pp. $260-278,2006$.

[16] A. Fall, A. Iggidr, G. Sallet, and J. J. Tewa, "Epidemiological models and Lyapunov functions," Mathematical Modelling of Natural Phenomena, vol. 2, no. 1, pp. 62-83, 2006.

[17] P. Adda, J. L. Dimi, A. Iggidr, J. C. a. Kamgang, and J. J. Tewa, "General models of host-parasite systems. Global analysis," Discrete and Continuous Dynamical Systems. Series B, vol. 8, no. 1, pp. 1-17, 2007.

[18] N. Bame, S. Bowong, J. Mbang, G. Sallet, and J. Tewa, "Global stability analysis for seis models with n latent classes," Mathematical Biosciences and Engineering, vol. 5, no. 1, pp. 20-23, 2008.

[19] H. I. Freedman and J. W. H. So, "Global stability and persistence of simple food chains," Mathematical Biosciences, vol. 76, no. 1, pp. 69-86, 1985.

[20] B. S. Goh, Management and Analysis of Biological Populations, Elsevier, Amsterdam, The Netherlands, 1980.

[21] S. Bowong and J. J. Tewa, "Mathematical analysis of a tuberculosis model with differential infectivity," Communications in Nonlinear Science and Numerical Simulation, vol. 14, no. 11, pp. 4010-4021, 2009.

[22] P. Van Den Driessche and J. Watmough, "Reproduction numbers and sub-threshold endemic equilibria for compartmental models of disease transmission," Mathematical Biosciences, vol. 180, pp. 29-48, 2002.

[23] J. J. Tewa, S. Bowong, and S. C. Oukouomi Noutchie, "Mathematical analysis of a two-patch model of tuberculosis disease with staged progression," Applied Mathematical Modelling, vol. 36, no. 12, pp. 5792-5807, 2012.

[24] A. Atangana and N. Bildik, "Approximate solution of tuberculosis disease population dynamics model," Abstract and Applied Analysis, vol. 2013, Article ID 759801, 8 pages, 2013.

[25] A. Atangana and E. Alabaraoye, "Solving a system of fractional partial differential equations arising in the model of HIV infection of $\mathrm{CD}^{+}$cells and attractor one-dimensional KellerSegel equations," Advances in Difference Equations, vol. 2013, article 94, 14 pages, 2013. 


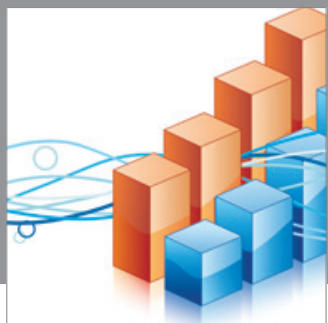

Advances in

Operations Research

mansans

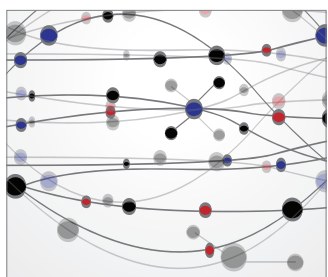

The Scientific World Journal
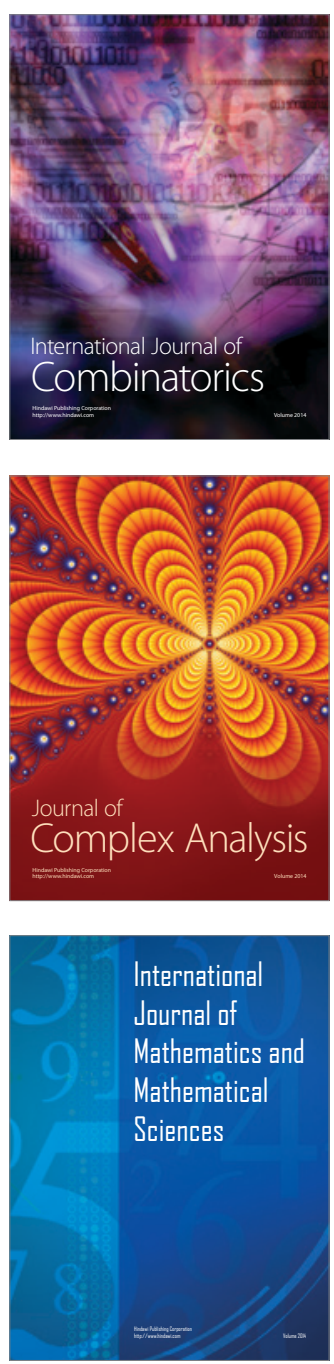
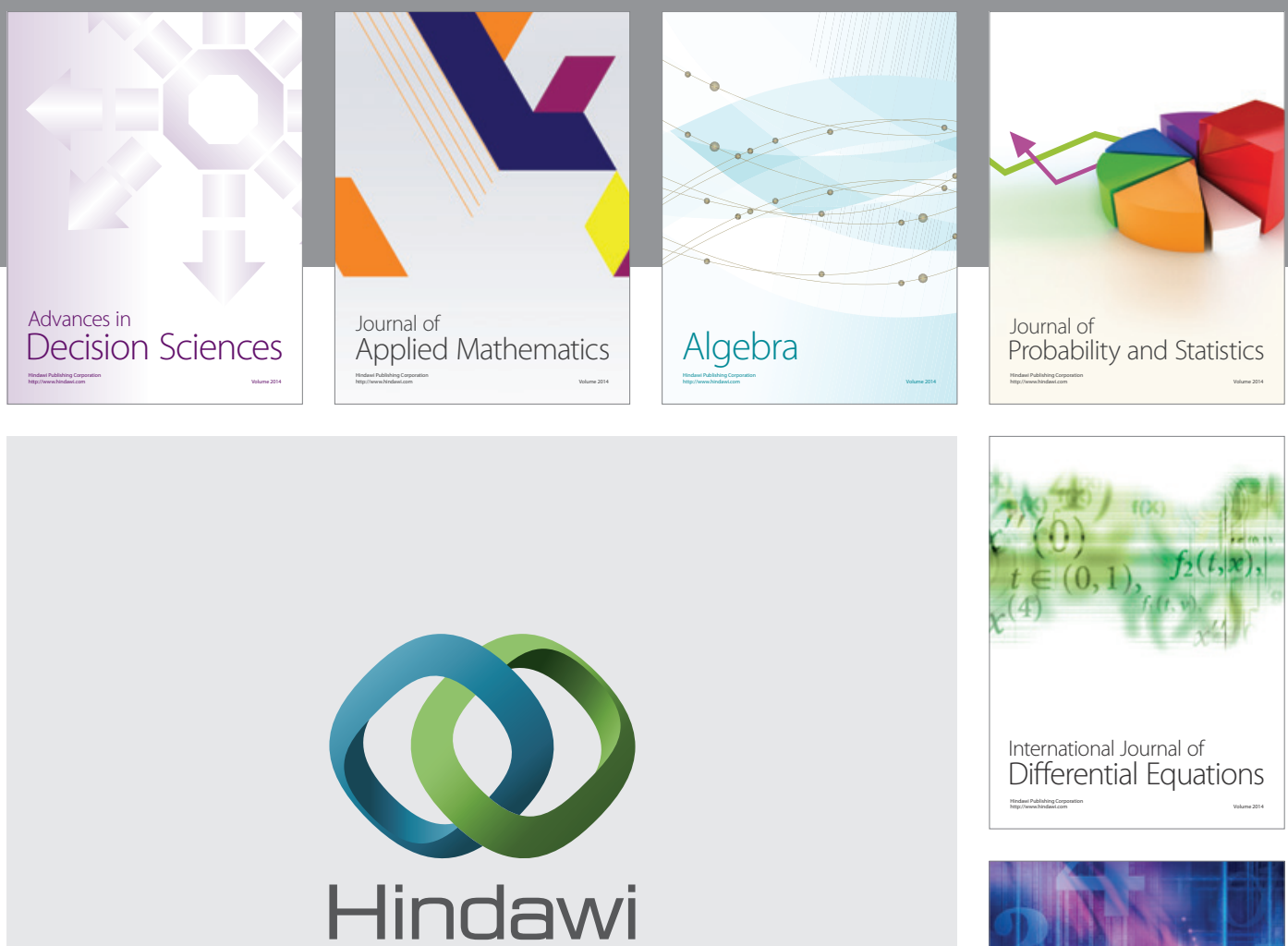

Submit your manuscripts at http://www.hindawi.com
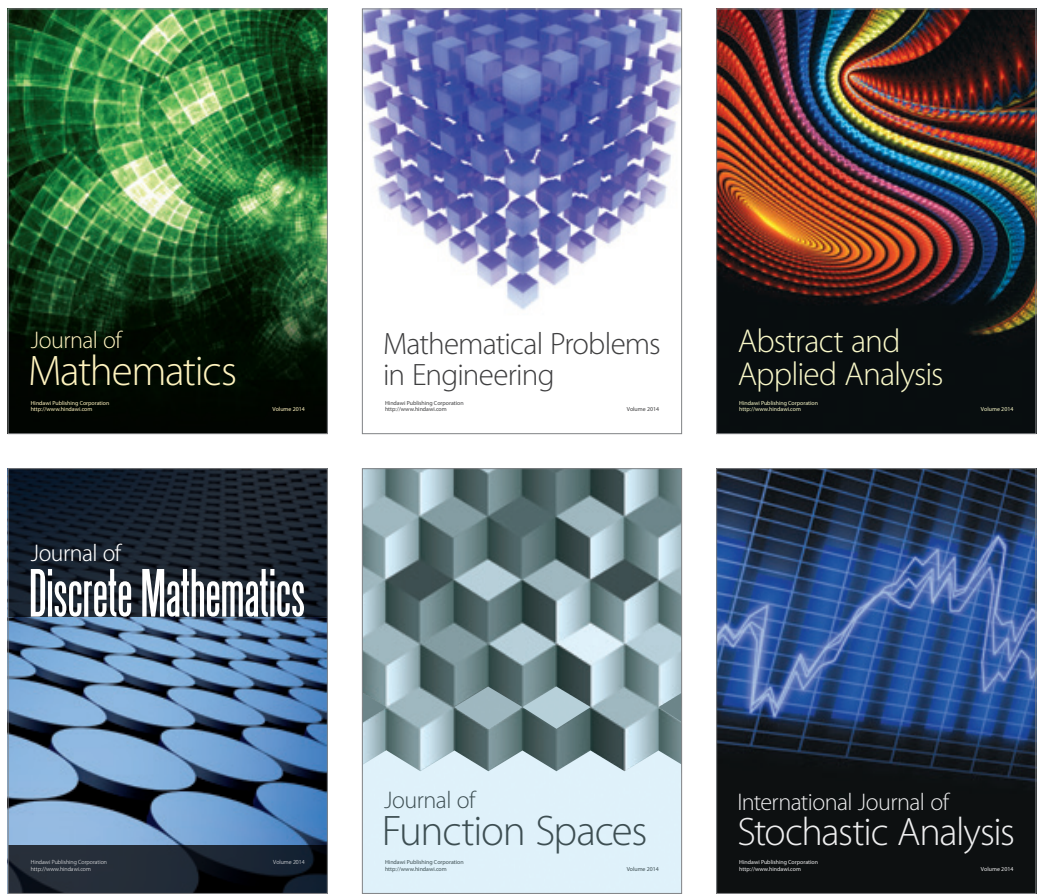

Journal of

Function Spaces

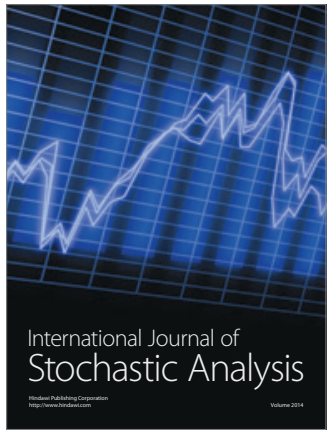

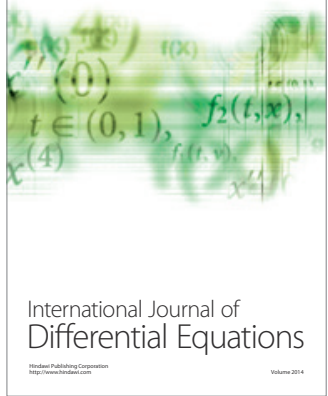
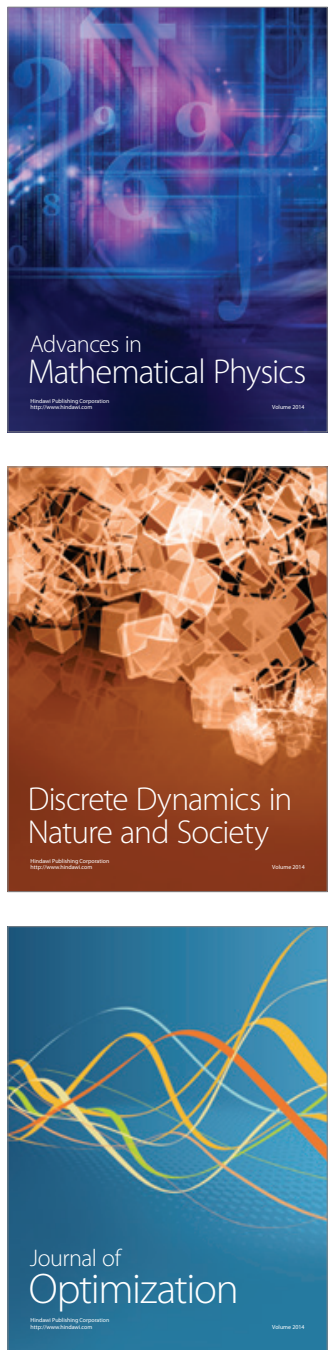